\title{
CONSTRUCTIONS OF COMMUTATIVE AUTOMORPHIC LOOPS
}

\author{
PŘEMYSL JEDLIČKA, MICHAEL K. KINYON, AND PETR VOJTĚCHOVSKÝ
}

\begin{abstract}
A loop whose inner mappings are automorphisms is an automorphic loop (or $A$-loop). We characterize commutative (A-)loops with middle nucleus of index 2 and solve the isomorphism problem. Using this characterization and certain central extensions based on trilinear forms, we construct several classes of commutative A-loops of order a power of 2. We initiate the classification of commutative A-loops of small orders and also of order $p^{3}$, where $p$ is a prime.
\end{abstract}

\section{INTRODUCTION}

A loop is a groupoid $(Q, \cdot)$ with neutral element 1 such that all left translations $L_{x}: Q \rightarrow Q$, $y \mapsto x y$ and all right translations $R_{x}: Q \rightarrow Q, y \mapsto y x$ are bijections of $Q$. Given a loop $Q$ and $x, y \in Q$, we denote by $x \backslash y$ the unique element of $Q$ satisfying $x(x \backslash y)=y$. In other words, $x \backslash y=L_{x}^{-1}(y)$.

To reduce the number of parentheses, we adopt the following convention for term evaluation: $\backslash$ is less binding than juxtaposition, and - is less binding than $\backslash$. For instance $x y \backslash u \cdot v \backslash w$ is parsed as $((x y) \backslash u)(v \backslash w)$.

The inner mapping group $\operatorname{Inn}(Q)$ of a loop $Q$ is the permutation group generated by

$$
L_{x, y}=L_{y x}^{-1} L_{y} L_{x}, \quad R_{x, y}=R_{x y}^{-1} R_{y} R_{x}, \quad T_{x}=L_{x}^{-1} R_{x},
$$

where $x, y \in Q$. A subloop of $Q$ is normal if it is invariant under all inner mappings of $Q$.

A loop $Q$ is an automorphic loop (or A-loop) if $\operatorname{Inn}(Q) \leq \operatorname{Aut}(Q)$, that is, if every inner mapping of $Q$ is an automorphism of $Q$. Hence a commutative loop is an A-loop if and only if all its left inner mappings $L_{y, x}$ are automorphisms, which can be expressed by the identity

$$
x y \backslash x(y u) \cdot x y \backslash x(y v)=x y \backslash x(y \cdot u v) .
$$

Note that the class of commutative A-loops contains commutative groups and commutative Moufang loops.

We assume that the reader is familiar with the terminology and notation of loop theory, cf. [1] or [10. This paper is a companion to [6], where we have presented a historical introduction and many new structural results concerning commutative $A$-loops, including:

- commutative A-loops are power-associative (see already [2]),

- for a prime $p$, a finite commutative A-loop $Q$ has order a power of $p$ if and only if every element of $Q$ has order a power of $p$,

- every finite commutative A-loop is a direct product of a loop of odd order (consisting of elements of odd order) and a loop of order a power of 2 ,

- commutative A-loops of odd order are solvable,

- the Lagrange and Cauchy theorems hold for commutative A-loops,

- every finite commutative A-loop has Hall $\pi$-subloops (and hence Sylow $p$-subloops),

2000 Mathematics Subject Classification. 20 N05.

Key words and phrases. commutative automorphic loop, commutative A-loop, automorphic inner mappings, central extensions, enumeration of A-loops.

Přemysl Jedlička supported by the Grant Agency of the Czech Republic, grant no. 201/07/P015. 
- if there is a nonassociative finite simple commutative A-loop, it is of exponent 2.

Despite these deep results, the theory of commutative A-loops is in its infancy. As an illustration of this fact, the present theory is not sufficiently developed to classify commutative A-loops of order 8 without the aid of a computer, commutative A-loops of order $p q$ (where $p<q$ are primes), nor commutative A-loops of order $p^{3}$ (where $p$ is an odd prime).

The two main problems for commutative A-loops stated in [6] were: For an odd prime $p$, is every commutative A-loop of order $p^{k}$ centrally nilpotent? Is there a nonassociative finite simple commutative A-loop, necessarily of exponent 2 and order a power of 2 ? For an example of a commutative A-loop of order 8 that is not centrally nilpotent, see Subsection 3.1.

In the meantime, we have managed to solve the first problem of [6] in the affirmative, but we neither use nor prove the result here - it will appear elsewhere. The second problem remains open and the many constructions of commutative A-loops of exponent 2 obtained here can be seen as a step toward solving it.

One of the most important concepts in the investigation of commutative A-loops appears to be the middle nucleus $N_{\mu}(Q)$, since, by [2], $N_{\lambda}(Q) \leq N_{\mu}(Q), N_{\rho}(Q) \leq N_{\mu}(Q)$ and $N_{\mu}(Q) \unlhd Q$ is true in any A-loop $Q$. In $₫ 2$ we characterize all commutative loops with middle nucleus of index 2, solve the isomorphism problem, and then characterize all commutative A-loops with middle nucleus of index 2. In 93 we classify commutative A-loops of order 8 , among other applications of 92

Central extensions of commutative A-loops are described in 4 A broad class of such extensions is obtained from trilinear forms that are symmetric with respect to an interchange of (fixed) two arguments. As an application, we characterize all parameters $(k, \ell)$ with the property that there is a nonassociative commutative A-loop of order $2^{k}$ with middle nucleus of order $2^{\ell}>1$.

\$5 uses another class of central extensions partially based on the overflow in modular arithmetic that yields many (conjecturally, all) nonassociative commutative A-loops of order $p^{3}$, where $p$ is an odd prime.

A classification of commutative A-loops of small orders based on the theory and computer computations can be found in 8 .

\section{Commutative loops With middle nucleus of IndeX 2}

Throughout this section, we denote by $\bar{X}=\{\bar{x} ; x \in X\}$ a disjoint copy of the set $X$.

Let $G$ be a commutative group and $f$ a bijection of $G$. Then $G(f)$ will denote the groupoid $(G \cup \bar{G}, *)$ with multiplication

$$
x * y=x y, \quad x * \bar{y}=\overline{x y}, \quad \bar{x} * y=\overline{x y}, \quad \bar{x} * \bar{y}=f(x y),
$$

for $x, y \in G$. Note that $G(f)$ is a loop with neutral element 1.

Lemma 2.1. Let $G$ be a commutative group, $f$ a bijection of $G$ and $(Q, \cdot)=G(f)=(G \cup \bar{G}, *)$. Then:

(i) $Q$ is commutative.

(ii) $x \backslash y=x^{-1} y, x \backslash \bar{y}=\overline{x^{-1} y}, \bar{x} \backslash y=\overline{x^{-1} f^{-1}(y)}, \bar{x} \backslash \bar{y}=x^{-1} y$ for every $x, y \in G$.

(iii) $G \leq N_{\mu}(Q)$.

(iv) $Q$ is a group if and only if $f$ is a translation of the group $G$.

(v) $N_{\lambda}(Q) \cap G=N_{\rho}(Q) \cap G=Z(Q) \cap G=\{x \in G ; f(x y)=x f(y)$ for every $y \in G\}$. When $Q$ is not a group (that is, $G=N_{\mu}(Q)$ ), then $N_{\lambda}(Q)=N_{\rho}(Q)=Z(Q) \leq G$.

Proof. Part (i) follows from the definition of $G(f)$. Part (ii) is straightforward, for instance, $x * \overline{x^{-1} y}=\overline{x x^{-1} y}=\bar{y}$ shows that $x \backslash \bar{y}=\overline{x^{-1} y}$. 
For (iii), let $x, y, z \in G$ and verify that

$$
\begin{aligned}
& x *(y * z)=(x * y) * z, \\
& \bar{x} *(y * z)=\bar{x} * y z=\overline{x y z}=\overline{x y} * z=(\bar{x} * y) * z, \\
& x *(y * \bar{z})=x * \overline{y z}=\overline{x y z}=x y * \bar{z}=(x * y) * \bar{z}, \\
& \bar{x} *(y * \bar{z})=\bar{x} * \overline{y z}=f(x y z)=\overline{x y} * \bar{z}=(\bar{x} * y) * \bar{z} .
\end{aligned}
$$

This shows $G \leq N_{\mu}(Q)$.

(iv) An easy calculation shows that $\overline{1} \in N_{\mu}(Q)$ (that is, $Q$ is a group) if and only if $f(x y)=x f(y)=f(x) y$ for every $x, y \in G$. With $y=1$ we deduce that $f(x)=x f(1)$ for every $x$. On the other hand, if $f(x)=x f(1)$ for every $x$ then $f(x y)=x f(y)=f(x) y$.

(v) We have $x *(y * z)=(x * y) * z, x *(\bar{y} * z)=\overline{x y z}=(x * \bar{y}) * z, x *(y * \bar{z})=\overline{x y z}=(x * y) * \bar{z}$, and $x *(\bar{y} * \bar{z})=x f(y z)$, while $(x * \bar{y}) * \bar{z}=f(x y z)$. Hence $x \in N_{\lambda}(Q)$ if and only if $x f(y z)=f(x y z)$ for every $y, z \in G$, which holds if and only if $x f(y)=f(x y)$ for every $y \in G$. By commutativity, $N_{\lambda}(Q)=N_{\rho}(Q)$. By (iii), $N_{\lambda}(Q) \cap G=Z(Q) \cap G$.

Assume that $Q$ is not a group. Suppose that $\bar{x} \in N_{\lambda}(Q)$. Then $f(x y z)=\bar{x} * \overline{y z}=\bar{x} *(\bar{y} * z)=$ $(\bar{x} * \bar{y}) * z=f(x y) * z=f(x y) z$ for every $y, z \in G$, and hence (with $\left.y=x^{-1}\right), f(z)=f(1) z$ for every $z \in G$. By (iv), $Q$ is a group, a contradiction. Thus $N_{\lambda}(Q) \leq G$.

Lemma 2.2. Let $Q$ be a commutative loop with subloop $G$ satisfying $G \leq N_{\mu}(Q),[Q: G]=2$. Then $G$ is a commutative group and there exists a bijection $f$ of $G$ such that $Q$ is isomorphic to $G(f)$.

Proof. The commutative loop $G$ is a group by $G \leq N_{\mu}(Q)$. Denote by $\overline{1}$ a fixed element of $Q \backslash G$, and define $\bar{x}=\overline{1} x=x \overline{1}$ for every $x \in G$. Note that $\overline{1}$ is well-defined, $G \cap \bar{G}=\emptyset$ and $Q=G \cup \bar{G}$. Moreover, $x \bar{y}=x \cdot y \overline{1}=x y \cdot \overline{1}=\overline{x y}$ and $\bar{x} y=\overline{1} x \cdot y=\overline{1} \cdot x y=\overline{x y}$ for every $x$, $y \in G$, using $G \leq N_{\mu}(Q)$ again. Finally, if $x_{1}, y_{1}, x_{2}, y_{2} \in G$ satisfy $x_{1} y_{1}=x_{2} y_{2}$ then

$$
\overline{x_{1} y_{1}}=\overline{1} x_{1} \cdot y_{1} \overline{1}=\overline{1}\left(x_{1} \cdot y_{1} \overline{1}\right)=\overline{1}\left(x_{1} y_{1} \cdot \overline{1}\right)=\overline{1}\left(x_{2} y_{2} \cdot \overline{1}\right)=\overline{x_{2} y_{2}} .
$$

Thus the multiplication in the quadrant $\bar{G} \times \bar{G}$ mimics that of $G \times G$, except that the elements are renamed according to the permutation $f: G \rightarrow G, x \mapsto \overline{1} \cdot x \overline{1}$.

Corollary 2.3. Let $Q$ be a commutative loop possessing a subgroup of index 2. Then $[Q$ : $\left.N_{\mu}(Q)\right] \leq 2$ if and only if there exists a commutative group $G$ and a bijection $f$ of $G$ such that $Q$ is isomorphic to $G(f)=(G \cup \bar{G}, *)$ defined by (2.1).

Remark 2.4. The assumption that $Q$ possesses a subgroup of index 2 in Corollary 2.3 is needed only when $Q$ is a group.

We now solve the isomorphism problem for nonassociative commutative loops with middle nucleus of index 2 in terms of the associated bijections:

Proposition 2.5. Let $G$ be a commutative group and $f_{1}, f_{2}$ bijections of $G$ such that $G\left(f_{1}\right)$, $G\left(f_{2}\right)$ are not groups. Then $G\left(f_{1}\right) \cong G\left(f_{2}\right)$ if and only if there is $\psi \in \operatorname{Aut}(G)$ such that

$$
f_{2}^{-1} \psi f_{1}(x)=f_{2}^{-1} \psi f_{1}(1) \cdot \psi(x) \quad \text { for all } x \in G,
$$

and $f_{2}^{-1} \psi f_{1}(1)$ is a square in $G$.

Proof. Denote by $*$ the multipication in $G\left(f_{1}\right)$, and by $\circ$ the multiplication in $G\left(f_{2}\right)$.

Assume that $\varphi: G\left(f_{1}\right) \rightarrow G\left(f_{2}\right)$ is an isomorphism. Since $G\left(f_{1}\right), G\left(f_{2}\right)$ are not groups, $\varphi$ maps $N_{\mu}\left(G\left(f_{1}\right)\right)=G$ onto $N_{\mu}\left(G\left(f_{2}\right)\right)=G$ by Lemma 2.1(iii), and hence $\psi=\left.\varphi\right|_{G}$ is a bijection of $G$. Then

$$
\psi(x y)=\varphi(x y)=\varphi(x * y)=\varphi(x) \circ \varphi(y)=\psi(x) \circ \psi(y)=\psi(x) \psi(y)
$$


for every $x, y \in G$, so $\psi \in \operatorname{Aut}(G)$.

Define $\rho: G \rightarrow G$ by $\overline{\rho(x)}=\varphi(\bar{x})$. We have

$$
\overline{\rho(x)}=\varphi(\bar{x})=\varphi(x * \overline{1})=\varphi(x) \circ \varphi(\overline{1})=\psi(x) \circ \overline{\rho(1)}=\overline{\psi(x) \rho(1)},
$$

so $\rho(x)=\rho(1) \psi(x)$ for every $x \in G$. Using this observation, we have

$\psi\left(f_{1}(x y)\right)=\varphi\left(f_{1}(x y)\right)=\varphi(\bar{x} * \bar{y})=\varphi(\bar{x}) \circ \varphi(\bar{y})=\overline{\rho(x)} \circ \overline{\rho(y)}=f_{2}(\rho(x) \rho(y))=f_{2}\left(\rho(1)^{2} \psi(x y)\right)$.

Equivalently, $f_{2}^{-1} \psi f_{1}(x)=\rho(1)^{2} \psi(x)$ for every $x \in G$. With $x=1$, we deduce that $\rho(1)^{2}=$ $f_{2}^{-1} \psi f_{1}(1)$ is a square in $G$, and that (2.2) holds.

Conversely, assume that (2.2) holds for some $\psi \in \operatorname{Aut}(G)$, and that $u^{2}=f_{2}^{-1} \psi f_{1}(1)$ is a square in $G$. Define $\varphi: G\left(f_{1}\right) \rightarrow G\left(f_{2}\right)$ by $\varphi(x)=\psi(x), \varphi(\bar{x})=\overline{u \psi(x)}$. Then

$$
\begin{gathered}
\varphi(x * y)=\varphi(x y)=\psi(x y)=\psi(x) \psi(y)=\psi(x) \circ \psi(y)=\varphi(x) \circ \varphi(y), \\
\varphi(\bar{x} * y)=\varphi(\overline{x y})=\overline{u \psi(x y)}=\overline{u \psi(x) \psi(y)}=\overline{u \psi(x)} \circ \psi(y)=\varphi(\bar{x}) \circ \varphi(y),
\end{gathered}
$$

and, similarly, $\varphi(x * \bar{y})=\varphi(x) \circ \varphi(\bar{y})$ for every $x, y \in G$. Finally, using (2.2) to obtain the third equality below, we have

$$
\varphi(\bar{x} * \bar{y})=\varphi\left(f_{1}(x y)\right)=\psi\left(f_{1}(x y)\right)=f_{2}\left(u^{2} \psi(x y)\right)=\overline{u \psi(x)} \circ \overline{u \psi(y)}=\varphi(\bar{x}) \circ \varphi(\bar{y})
$$

for every $x, y \in G$. Thus $G\left(f_{1}\right) \cong G\left(f_{2}\right)$.

We say that two bijections $f_{1}, f_{2}$ of $G$ are conjugate in $\operatorname{Aut}(G)$ if there is $\psi \in \operatorname{Aut}(G)$ such that $f_{2}=\psi f_{1} \psi^{-1}$. The following specialization of Proposition 2.5 will be useful in the classification of commutative A-loops of order 8.

Corollary 2.6. Let $G$ be a commutative group, and let $f_{1}, f_{2}$ be bijections of $G$ such that $G\left(f_{1}\right), G\left(f_{2}\right)$ are not groups.

(i) If $f_{1}, f_{2}$ are conjugate in $\operatorname{Aut}(G)$ then $G\left(f_{1}\right) \cong G\left(f_{2}\right)$.

(ii) If $f_{1}(1)=1=f_{2}(1)$ then $G\left(f_{1}\right) \cong G\left(f_{2}\right)$ if and only if $f_{1}, f_{2}$ are conjugate in Aut $(G)$.

(iii) If $f_{2} \in \operatorname{Aut}(G), t$ is a square in $G$ and $f_{1}(x)=f_{2}(x) t$ for every $x \in G$ then $G\left(f_{1}\right) \cong$ $G\left(f_{2}\right)$.

Proof. (i) Let $\psi \in \operatorname{Aut}(G)$ be such that $f_{2}=\psi f_{1} \psi^{-1}$. Then $f_{2}^{-1} \psi f_{1}=\psi$, so $f_{2}^{-1} \psi f_{1}(1)=$ $\psi(1)=1$ is a square in $G$ and (2.2) holds.

(ii) Assume that $G\left(f_{1}\right) \cong G\left(f_{2}\right)$. Then there is $\psi \in \operatorname{Aut}(G)$ such that (2.2) holds. Since $f_{2}^{-1} \psi f_{1}(1)=f_{2}^{-1} \psi(1)=f_{2}^{-1}(1)=1$, we deduce from (2.2) that $f_{1}, f_{2}$ are conjugate in $\operatorname{Aut}(G)$. The converse follows by (i).

(iii) Let $\psi$ be the trivial automorphism of $G$. Then (2.2) becomes $f_{2}^{-1} f_{1}(x)=f_{2}^{-1} f_{1}(1) \cdot x$, and it is our task to check this identity and that $f_{2}^{-1} f_{1}(1)$ is a square in $G$. Now, $f_{2}^{-1} f_{1}(1)=$ $f_{2}^{-1}\left(f_{2}(1) t\right)=f_{2}^{-1}\left(f_{2}(1)\right) f_{2}^{-1}(t)=f_{2}^{-1}(t)$ is a square in $G$ since $t$ is. Moreover, $f_{1}(1)=$ $f_{2}(1) \cdot t=t$, so $f_{1}(x)=f_{1}(1) f_{2}(x)$, and (2.2) follows upon applying $f_{2}^{-1}$ to this equality.

Finally, we describe all commutative A-loops with middle nucleus of index 2 .

Proposition 2.7. Let $Q$ be a commutative loop possessing a subgroup of index 2 . Then the following conditions are equivalent:

(i) $Q$ is an $A$-loop and $\left[Q: N_{\mu}(Q)\right] \leq 2$. 
(ii) $Q=G(f)$, where $G$ is a commutative group, $[Q: G]=2$, and $f$ is a permutation of $G$ satisfying

$$
\begin{aligned}
& f(x y)=f(x) f(y) f(1)^{-1}, \\
& f\left(x^{2}\right)=x^{2} f(1) \\
& f^{2}(x)^{2} f(x)^{-2}=f^{2}(1)
\end{aligned}
$$

for every $x, y \in G$.

(iii) $Q=G(f)$, where $G$ is a commutative group, $[Q: G]=2$, and $f$ is a permutation of $G$ satisfying $\left(P_{1}\right),\left(P_{2}\right)$ and $f^{2}(1)=f(1)^{2}$.

(iv) $Q=G(f)$, where $G$ is a commutative group, $[Q: G]=2, f(x)=g(x) t$ for every $x \in G, g \in \operatorname{Aut}(G), g\left(x^{2}\right)=x^{2}$ for every $x \in G$, and $t$ is a fixed point of $g$.

Proof. By Corollary 2.3, we can assume that $Q=G(f)=(G \cup \bar{G}, *)$, where $G \leq N_{\mu}(Q)$ is a commutative group and $f$ is a bijection of $G$. Let us establish the equivalence of (i) and (ii).

Denote by $\alpha(a, b, c, d)$ the $*$ version of (A) , namely

$$
(a * b) \backslash(a *(b *(c * d)))=[(a * b) \backslash(a *(b * c))] *[(a * b) \backslash(a *(b * d))],
$$

where $a, b, c, d$ are taken from $G \cup \bar{G}$, and where $\backslash$ is understood in $(Q, *)$. With the exception of the variables $a, b, c, d$, we implicitly assume that variables without bars are taken from $G$, while variables with bars are taken from $\bar{G}$.

Then $\alpha(x, y, u, v)$ holds in $G(f)$, as the evaluation of $\alpha(x, y, u, v)$ takes place in the group $G$. Since $y \in N_{\mu}(Q), \alpha(a, y, c, d)$ holds. By commutativity of $*, \alpha(a, b, c, d)$ holds if and only if $\alpha(a, b, d, c)$ holds. Hence it remains to investigate the identities $\alpha(x, \bar{y}, u, v), \alpha(x, \bar{y}, u, \bar{v})$, $\alpha(x, \bar{y}, \bar{u}, \bar{v}), \alpha(\bar{x}, \bar{y}, u, v), \alpha(\bar{x}, \bar{y}, u, \bar{v})$, and $\alpha(\bar{x}, \bar{y}, \bar{u}, \bar{v})$.

Straightforward calculation with (2.1) and Lemma 2.1 shows that $\alpha(\bar{x}, \bar{y}, u, \bar{v})$ holds if and only if

$$
f(y u v)=f(x y)^{-1} f(x y u) f(y v) .
$$

Using $x=y=1$, (2.3) reduces to $\left(P_{1}\right)$. On the other hand, $\left(P_{1}\right)$ already implies (2.3), and so $\alpha(\bar{x}, \bar{y}, u, \bar{v})$ is equivalent to $\left(P_{1}\right)$. From now on, we will assume that $\left(P_{1}\right)$ holds and denote $f(1)$ by $t$.

The identity $\alpha(x, \bar{y}, \bar{u}, \bar{v})$ is then equivalent to

$$
x^{-1} t^{-1}=f\left(x^{-2}\right) f\left(y^{-2}\right) f(y)^{2} x t^{-5},
$$

and since $t=f\left(y y^{-1}\right)=f(y) f\left(y^{-1}\right) t^{-1}$ yields

$$
f\left(y^{-1}\right)=f(y)^{-1} t^{2}
$$

we can rewrite (2.4) as $f(x)^{2}=x^{2} t^{2}$, or, equivalently (using (Pי $)$ ), as $\left(P_{2}\right)$.

Finally, note that $\left(P_{1}\right)$ and $(2.5)$ imply

$$
f^{2}(u v)=f(f(u v))=f\left(f(u) f(v) t^{-1}\right)=f^{2}(u) f^{2}(v) f\left(t^{-1}\right) t^{-2}=f^{2}(u) f^{2}(v) f(t)^{-1} .
$$

Using (2.6) and (2.5), we see, after a lengthy calculation, that the identity $\alpha(\bar{x}, \bar{y}, \bar{u}, \bar{v})$ is equivalent to $\left(P_{3}\right)$.

We leave it to the reader to check that the identities $\alpha(x, \bar{y}, u, v), \alpha(x, \bar{y}, u, \bar{v}), \alpha(x, \bar{y}, \bar{u}, \bar{v})$ imply no additional conditions on $f$ beside $\left(\overline{P_{1}}-\left(\overline{P_{3}}\right)\right.$, and, conversely, that if $\left(P_{1}\right)-\left(P_{3}\right)$ are satisfied then the identities $\alpha(x, \bar{y}, u, v), \alpha(x, \bar{y}, u, \bar{v}), \alpha(x, \bar{y}, \bar{u}, \bar{v})$ hold.

We have proved the equivalence of (i) and (ii).

Assume that (ii) holds. With $x=1$ in $\left(\overline{P_{3}}\right)$ we have $f^{2}(1)^{2} f(1)^{-2}=f(t)$, or $f(t)^{2} t^{-2}=f(t)$, or $f(t)=t^{2}$, so (iii) holds. Conversely, assume that (iii) holds. Then, $f^{2}(x)^{2} f(t)^{-1}=$ $f^{2}(x)^{2} t^{-2}=f(f(x)) f(f(x)) t^{-2}=f(f(x) f(x)) t^{-1}=f\left(f(x)^{2}\right) t^{-1}=f(x)^{2}$, which is (Pי), so (ii) holds. 
Assume that (iii) holds and define $g$ by $g(x)=f(x) t^{-1}$, where $t=f(1)$. Then $g(x y)=$ $f(x y) t^{-1}=f(x) f(y) t^{-2}=f(x) t^{-1} f(y) t^{-1}=g(x) g(y)$ by $\left(P_{1}\right), g\left(x^{2}\right)=f\left(x^{2}\right) t^{-1}=x^{2}$ by $\left(P_{2}\right)$, and $g(t)=f(t) t^{-1}=t$ by $f(t)=t^{2}$. Conversely, assume that (iv) holds, $f(x)=g(x) t$, $g \in \operatorname{Aut}(G)$, where $g\left(x^{2}\right)=x^{2}$ and $t$ is a fixed point of $g$ (not necessarily satisfying $t=f(1)$ ). Then $f(1)=g(1) t=t, f(x y)=g(x y) t=g(x) g(y) t=g(x) t g(y) t t^{-1}=f(x) f(y) t^{-1}, f\left(x^{2}\right)=$ $g\left(x^{2}\right) t=x^{2} t$, and $f(t)=g(t) t=t^{2}$, proving (iii).

\section{Constructions of Commutative A-Loops with middle nucleus of index 2}

As an application of Proposition 2.7, we classify all commutative A-loops of order 8 and present a class of commutative A-loops of exponent 2 with trivial center and middle nucleus of index 2 .

3.1. Commutative A-loops of order 8. It is not difficult to classify all commutative Aloops of order 8 up to isomorphism with a finite model builder, such as Mace4 [7]. It turns out that there are 4 nonassociative commutative A-loops of order 8. All such loops have middle nucleus of index 2; a fact for which we do not have a human proof. But using this fact, we can finish the classification by hand with Proposition 2.5, Corollary 2.6 and Proposition 2.7.

Lemma 3.1. Let $G$ be a commutative loop, $1 \neq g \in \operatorname{Aut}(G)$ and $t \in G$. Let $f$ be a bijection of $G$ defined by $f(x)=g(x) t$. Then $Z(G(f))=Z(G(g))$ as sets, and $Z(G(g))=\{x \in G ; g(x)=$ $x\}$.

Proof. Since $g$ is not a translation of $G$, neither is $f$. Hence both $G(g)$ and $G(f)$ are nonassociative, by Lemma 2.1(iv). By Lemma 2.1(v), $Z(G(f))=\{x \in G ; f(x y)=x f(y)$ for every $y \in$ $G\}=\{x \in G ; g(x y) t=x g(y) t$ for every $y \in G\}=\{x \in G ; g(x y)=x g(y)$ for every $y \in G\}=$ $Z(G(g))$ and it is also equal to $\{x \in G ; g(x)=x\}$ since $g(x y)=g(x) g(y)$.

Let $Q$ be a nonassociative commutative A-loop of order 8 , necessarily with a middle nucleus of index 2. By Proposition 2.7. $Q=G(f)$, where $G$ is a commutative group of order 4 and $f(x)=g(x) t$ for some $g \in \operatorname{Aut}(G)$ and $t \in G$ such that $g\left(x^{2}\right)=x^{2}$ and $g(t)=t$.

Let $G=\mathbb{Z}_{4}=\langle a\rangle$ be the cyclic group of order 4 . The two automorphisms of $G$ are the trivial automorphism $g=1$ and the transposition $g=\left(a, a^{3}\right)$; both fix all squares of $G$. Let $g=1$ and $f(x)=g(x) t=x t$ for some $t \in G$. Then $G(f)$ is a commutative group by Lemma 2.1(iv). Assume that $g=\left(a, a^{3}\right)$. Then $G(g)$ is a nonassociative commutative A-loop. The only nontrivial fixed point of $g$ is $a^{2}$. Let $f(x)=g(x) a^{2}$. By Corollary 2.6(iii), $G(f) \cong G(g)$.

Now let $G=\mathbb{Z}_{2} \times \mathbb{Z}_{2}=\langle a\rangle \times\langle b\rangle$ be the Klein group. Then $\operatorname{Aut}(G)=\{1,(a, b),(a, a b)$, $(b, a b),(a, b, a b),(a, a b, b)\} \cong S_{3}$. The only square in $G$ is 1 and it is trivially fixed by all $g \in \operatorname{Aut}(G)$.

If $g=1$ and $f(x)=g(x) t=x t$ for some $t \in G, G(g)$ is a commutative group by Lemma 2.1(iv). Let $g_{1}=(a, b)$. The choices for $t$ are $t=1, t=a b$. Let $f_{1}(x)=g_{1}(x) a b$. Then $G\left(g_{1}\right)$, $G\left(f_{1}\right)$ are nonassociative commutative A-loops. Since $g_{1}(x x)=g_{1}(1)=1, G\left(g_{1}\right)$ has exponent 2. Since $f_{1}(x x)=f_{1}(1)=a b, G\left(f_{1}\right)$ does not have exponent 2 . Hence $G\left(g_{1}\right) \not G\left(f_{1}\right)$.

Let $g_{2}=(a, a b)$, and note that the choices for $t$ are $t=1, t=b$. Let $f_{2}(x)=g_{2}(x) b$. Since all transpositions of $S_{3}$ are conjugate in $S_{3}, G\left(g_{1}\right) \cong G\left(g_{2}\right)$ by Corollary [2.6(i). Note that $f_{1}=\psi^{-1} f_{2} \psi$ with $\psi=(b, a b)$. Hence $G\left(f_{1}\right) \cong G\left(f_{2}\right)$ by Corollary 2.6. Similarly, no new nonassociative commutative A-loop of order 8 is obtained with $g_{3}=(b, a b)$.

Let $g_{4}=(a, b, a b)$. Then $t=1$ is the only choice, and $G\left(g_{4}\right)$ is a nonassociative commutative A-loop. By Lemma 3.1, $Z\left(G\left(g_{4}\right)\right)=1$ and $Z\left(G\left(f_{1}\right)\right)=Z\left(G\left(g_{1}\right)\right) \cong \mathbb{Z}_{2}$. Thus $G\left(g_{4}\right)$ is a new nonassociative commutative A-loop. Finally, let $g_{5}=(a, a b, b)$. Since $g_{4}, g_{5}$ are conjugate in $\operatorname{Aut}(G), G\left(g_{4}\right) \cong G\left(g_{5}\right)$ by Corollary $2.6(\mathrm{i})$. 
3.2. A class of commutative A-loops of exponent 2 with trivial center and middle nucleus of index 2. Let $\operatorname{GF}(2)$ be the two-element field and let $V$ be a vector space over $\mathrm{GF}(2)$ of dimension $n \geq 2$. Let $G=(V,+)$ be the corresponding elementary abelian 2-group.

Let $\left\{e_{1}, \ldots, e_{n}\right\}$ be a basis of $V$. Define an automorphism of $G$ by

$$
g\left(e_{1}\right)=e_{2}, \quad g\left(e_{2}\right)=e_{3}, \quad g\left(e_{n-1}\right)=e_{n}, \quad g\left(e_{n}\right)=e_{1}+e_{n} .
$$

Since $g(x+x)=g(0)=0=g(x)+g(x)$, the equivalence of (i) and (iv) in Proposition 2.7 with $f=g$ shows that $Q_{n}=G(f)$ is a commutative A-loop of order $2^{n+1}$ with nucleus of index at most 2 .

We claim that $g$ has no fixed points besides 0 . Indeed, for $x=\sum_{i=1}^{n} \alpha_{i} e_{i}$ we have

$$
g(x)=\alpha_{n} e_{1}+\alpha_{1} e_{2}+\cdots \alpha_{n-2} e_{n-1}+\left(\alpha_{n-1}+\alpha_{n}\right) e_{n},
$$

so $x=g(x)$ if and only if

$$
\alpha_{1}=\alpha_{n}, \quad \alpha_{2}=\alpha_{1}, \quad \alpha_{n-1}=\alpha_{n-2}, \quad \alpha_{n}=\alpha_{n-1}+\alpha_{n},
$$

or, $\alpha_{1}=\cdots=\alpha_{n}=0$.

Thus Lemma 3.1 implies that $Z\left(Q_{n}\right)=1$, and $\left[Q_{n}: N_{\mu}\left(Q_{n}\right)\right]=2$ follows. Finally, $x * x=$ $x+x=0$ and $\bar{x} * \bar{x}=g(x+x)=0$ for every $x \in G$, so $Q_{n}$ has exponent two.

\section{Central extensions based on trilinear forms}

Let $Z, K$ be loops. We say that a loop $Q$ is an extension of $Z$ by $K$ if $Z \unlhd Q$ and $Q / Z \cong K$. If $Z \leq Z(Q)$, the extension is said to be central.

It is well-known that central extensions of an abelian group $Z$ by a loop $K$ are precisely the loops $K \ltimes_{\theta} Z$ defined on $K \times Z$ by

$$
(x, a)(y, b)=(x y, a b \theta(x, y)),
$$

where $\theta: K \times K \rightarrow Z$ is a (loop) cocycle, that is, a mapping satisfying $\theta(x, 1)=\theta(x, 1)=1$ for every $x \in K$.

In [2, Theorem 6.4], Bruck and Paige described all central extensions of an abelian group $Z$ by an A-loop $K$ resulting in an A-loop $Q$. The cocycle identity they found is rather complicated, and despite some optimism of Bruck and Paige, it is by no means easy to construct cocycles that conform to it.

In the commutative case, we deduce from [2, Theorem 6.4]:

Corollary 4.1. Let $Z$ be an abelian group and $K$ a commutative A-loop. Let $\theta: K \times K \rightarrow Z$ be a cocycle satisfying $\theta(x, y)=\theta(y, x)$ for every $x, y \in K$ and

$$
F(x, y, z) F\left(x^{\prime}, y, z\right) \theta\left(R_{y, z}(x), R_{y, z}\left(x^{\prime}\right)\right)=F\left(x x^{\prime}, y, z\right) \theta\left(x, x^{\prime}\right)
$$

for every $x, y, z, x^{\prime} \in K$, where

$$
F(x, y, z)=\theta\left(R_{y, z}(x), y z\right)^{-1} \theta(y, z)^{-1} \theta(x y, z) \theta(x, y) .
$$

Then $K \ltimes_{\theta} Z$ is a commutative A-loop.

Conversely, every commutative A-loop that is a central extension of $Z$ by $K$ can be represented in this manner.

Corollary 4.2. Let $Z$ be an elementary abelian 2-group and $K$ a commutative A-loop of exponent two. Let $\theta: K \times K \rightarrow Z$ be a cocycle satisfying $\theta(x, y)=\theta(y, x)$ for every $x, y \in K$, $\theta(x, x)=1$ for every $x \in K$, and

$$
\begin{aligned}
& \theta(x, y) \theta\left(x^{\prime}, y\right) \theta\left(x x^{\prime}, y\right) \theta\left(x, x^{\prime}\right) \theta(x y, z) \theta\left(x^{\prime} y, z\right) \theta(y, z) \theta\left(\left(x x^{\prime}\right) y, z\right)= \\
& \theta\left(R_{y, z}(x), y z\right) \theta\left(R_{y, z}\left(x^{\prime}\right), y z\right) \theta\left(R_{y, z}\left(x x^{\prime}\right), y z\right) \theta\left(R_{y, z}(x), R_{y, z}\left(x^{\prime}\right)\right)
\end{aligned}
$$


for every $x, y, z, x^{\prime} \in K$. Then $K \ltimes_{\theta} Z$ is a commutative A-loop of exponent two.

Conversely, every commutative A-loop of exponent two that is a central extension of $Z$ by $K$ can be represented in this manner.

When $K$ is an elementary abelian 2-group, the cocycle identity (4.2) can be rewritten as

$$
\begin{aligned}
& \theta(x, y) \theta\left(x^{\prime}, y\right) \theta\left(x x^{\prime}, y\right) \\
& \theta(x y, z) \theta\left(x^{\prime} y, z\right) \theta\left(x x^{\prime}, z\right) \\
& \theta(x, y z) \theta\left(x^{\prime}, y z\right) \theta\left(x x^{\prime}, y z\right) \\
& \theta(y, z) \theta\left(x x^{\prime}, z\right) \theta\left(\left(x x^{\prime}\right) y, z\right)=1 .
\end{aligned}
$$

Since every line above is of the form $\theta(u, w) \theta(v, w) \theta(u v, w)$, it is tempting to try to satisfy (4.2) by imposing $\theta(u, w) \theta(v, w) \theta(u v, w)=1$ for every $u, v, w \in K$. However, that identity already implies associativity. A nontrivial solution to the cocycle identity for commutative A-loops of exponent two can be obtained as follows:

Proposition 4.3. Let $Z=\mathrm{GF}(2)$ and let $K$ be an elementary abelian 2-group. Let $g$ : $K^{3} \rightarrow \mathrm{GF}(2)$ be a trilinear form such that $g(x, x y, y)=g(y, x y, x)$ for every $x, y \in K$. Define $\theta: K^{2} \rightarrow \mathrm{GF}(2)$ by $\theta(x, y)=g(x, x y, y)$. Then $Q=K \ltimes_{\theta} Z$ is a commutative Aloop of exponent 2. Moreover, $(y, b) \in N_{\mu}(Q)$ if and only if for every $x, z \in K$ we have $g(y, x, z)=g(x, z, y)$.

Proof. Trilinearity alone implies that $\theta(u, w) \theta(v, w) \theta(u v, w)=g(u, v, w) g(v, u, w)$. The lefthand side of (4.3) can then be rewritten as

$$
g\left(x, x^{\prime}, y\right) g\left(x^{\prime}, x, y\right) g\left(x y, x^{\prime} y, z\right) g\left(x^{\prime} y, x y, z\right) g\left(x, x^{\prime}, y z\right) g\left(x^{\prime}, x, y z\right) g\left(y, x x^{\prime}, z\right) g\left(x x^{\prime}, y, z\right),
$$

which reduces to 1 by trilinearity.

We have $(y, b) \in N_{\mu}(Q)$ if and only if $\theta(x, y) \theta(x y, z)=\theta(y, z) \theta(x, y z)$ for every $x, z \in K$, and the rest follows from trilinearity of $g$.

Let $V=\mathrm{GF}(2)^{n}$. Call a 3-linear form $g: V \rightarrow \mathrm{GF}(2)(1,3)$-symmetric if $g(x, y, z)=$ $g(z, y, x)$ for every $x, y, z \in V$. By Proposition 4.3, a $(1,3)$-symmetric trilinear form gives rise to a commutative A-loop $Q$ of exponent 2, and $(y, b) \in N_{\mu}(Q)$ if and only if $g(y, x, z)=$ $g(y, z, x)$ for every $x, z$, that is, if and only if the induced bilinear form $g(y,-,-): V^{2} \rightarrow \mathrm{GF}(2)$ is symmetric.

Lemma 4.4. Let $V$ be a vector space over $\mathrm{GF}(2)$ of dimension at least 3 . Then there exists a trilinear form $g: V \rightarrow \mathrm{GF}(2)$ such that for any $0 \neq x \in V$ the induced bilinear form $g(x,-,-): V^{2} \rightarrow \mathrm{GF}(2)$ is not symmetric.

Proof. Let $\left\{e_{1}, \ldots, e_{n}\right\}$ be a basis of $V$. The trilinear form $g$ is determined by the values $g\left(e_{i}, e_{j}, e_{k}\right) \in \mathrm{GF}(2)$, for $1 \leq i, j, k \leq n$. Set $g\left(e_{i}, e_{i}, e_{i+1}\right)=1$ for every $i$ (with $e_{n+1}=e_{1}$ ) and $g\left(e_{i}, e_{j}, e_{k}\right)=0$ otherwise.

Let $x=\sum \alpha_{j} e_{j}$ be such that $\alpha_{i} \neq 0$ for some $i$. Then $g\left(x, e_{i}, e_{i+1}\right)=\sum \alpha_{j} g\left(e_{j}, e_{i}, e_{i+1}\right)=$ $\alpha_{i} g\left(e_{i}, e_{i}, e_{i+1}\right)=\alpha_{i} \neq 0$, while, similarly, $g\left(x, e_{i+1}, e_{i}\right)=0$.

Example 4.5. By Lemma 4.4, for every $n \geq 3$ there is a commutative A-loop $Q$ of exponent 2 and order $2^{n+1}$ with $N_{\mu}(Q)=Z(Q),|Z(Q)|=2$.

Let $Q$ be a finite commutative A-loop of exponent 2. By results of $[6],|Q|=2^{k}$ for some $k$. Let $\left|N_{\mu}(Q)\right|=2^{\ell}$. We show how to realize all possible pairs $(k, \ell)$ with $\ell>0$.

Lemma 4.6. Let $k \geq \ell>0$. Then there is a nonassociative commutative A-loop of order $2^{k}$ with middle nucleus of order $2^{\ell}$ if and only if: either $d=k-\ell \geq 3$, or $d \geq 1$ and $\ell \geq 2$. 
Proof. If $d \geq 3$, consider the loop $Q$ of order $2^{d+1}$ with middle nucleus of order 2 from Example 4.5. Then $Q \times\left(\mathbb{Z}_{2}\right)^{k-(d+1)}$ achieves the parameters $(k, \ell)$.

Assume that $d=2$. The parameters $(3,1)$ are not possible by 93 , and the parameters $(4,2)$ are possible (see $₫ 6)$. Then $(k, \ell)$ can be achieved using the appropriate direct product.

Finally, assume that $d=1$. Then we are done by Subsection 3.2. We obviously must have $\ell \geq 2$, else $|Q|=2^{k} \leq 4$.

We remark that Lemma 4.4 cannot be improved:

Lemma 4.7. Let $V=\mathrm{GF}(2)^{n}$ and let $g: V^{3} \rightarrow \mathrm{GF}(2)$ be a (1,3)-symmetric trilinear form. If $n<3$ then there is $0 \neq x \in V$ such that the induced form $g(x,-,-)$ is symmetric.

Proof. There is nothing to show when $n=1$, so assume that $n=2$ and $\left\{e_{1}, e_{2}\right\}$ is a basis of $V$. The form $g$ is determined by the 6 values $g\left(e_{1}, e_{1}, e_{1}\right), g\left(e_{1}, e_{1}, e_{2}\right), g\left(e_{1}, e_{2}, e_{1}\right), g\left(e_{1}, e_{2}, e_{2}\right)$, $g\left(e_{2}, e_{1}, e_{2}\right)$ and $g\left(e_{2}, e_{2}, e_{2}\right)$.

Suppose that no induced form $g(x,-,-)$ is symmetric, for $0 \neq x \in V$. Then $g\left(e_{1}, e_{1}, e_{2}\right) \neq$ $g\left(e_{1}, e_{2}, e_{1}\right)$, else $g\left(e_{1},-,-\right)$ is symmetric. Similarly, $g\left(e_{2}, e_{1}, e_{2}\right) \neq g\left(e_{2}, e_{2}, e_{1}\right)$. But then $g\left(e_{1}+e_{2}, e_{1}, e_{2}\right)=g\left(e_{1}, e_{1}, e_{2}\right)+g\left(e_{2}, e_{1}, e_{2}\right)=g\left(e_{1}, e_{2}, e_{1}\right)+g\left(e_{2}, e_{2}, e_{1}\right)=g\left(e_{1}+e_{2}, e_{2}, e_{1}\right)$, hence $g\left(e_{1}+e_{2},-,-\right)$ is symmetric, a contradiction.

Remark 4.8. The many examples presented so far might suggest that $Q / N_{\mu}(Q)$ is a group in every commutative A-loop. This is not so: Consider a commutative Moufang loop $Q$. Then $Q$ is a commutative $A$-loop, and $N_{\mu}(Q)=Z(Q)$ since the three nuclei of $Q$ coincide. So the statement " $Q / N_{\mu}(Q)$ is a group" is equivalent to " $Q / Z(Q)$ is an abelian group", i.e., to " $Q$ has nilpotency class at most 2". There are commutative Moufang loops of nilpotency class 3.

Problem 4.9. Find a smallest commutative A-loop $Q$ in which $Q / N_{\mu}(Q)$ is not a group.

4.1. Adding group cocycles. Let $Z$ be an abelian group and $K$ a loop. Then a loop cocycle $\theta: K \times K \rightarrow Z$ is said to be a group cocycle if it satisfies the identity

$$
\theta(x, y) \theta(x y, z)=\theta(y, z) \theta(x, y z) .
$$

Note that if $K$ is a group and $\theta$ is a group cocycle then $K \ltimes_{\theta} Z$ is a group, too.

Lemma 4.10. Let $Z$ be an abelian group, $K$ a group and $\theta, \mu: K \times K \rightarrow Z$ loop cocycles such that $\nu=\theta \mu^{-1}:(x, y) \mapsto \theta(x, y) \mu(x, y)^{-1}$ is a group cocycle. Then the left inner mappings in $K \ltimes_{\theta} Z$ and $K \ltimes_{\mu} Z$ coincide.

Proof. Calculating in $K \ltimes_{\theta} Z$, we have

$$
\begin{aligned}
(x, a)(y, b) & =(x y, a b \theta(x, y)), \\
(x, a) \backslash(y, b) & =\left(x \backslash y, a^{-1} b \theta(x, x \backslash y)^{-1}\right) .
\end{aligned}
$$

Then

$$
\begin{aligned}
(x, a)(y, b) \backslash(x, a)((y, b)(z, c))=(x y, a b \theta(x, y)) \backslash(x y z, a b c \theta(x, y z) \theta(y, z) & \\
& =\left(z, c \theta(x, y z) \theta(y, z) \theta(x, y)^{-1} \theta(x y, z)^{-1}\right) .
\end{aligned}
$$

Thus the left inner mappings in $K \ltimes_{\theta} Z$ and $K \ltimes_{\mu} Z$ coincide if and only if

$$
\theta(x, y z) \theta(y, z) \theta(x, y)^{-1} \theta(x y, z)^{-1}=\mu(x, y z) \mu(y, z) \mu(x, y)^{-1} \mu(x y, z)^{-1}
$$

for every $x, y, z \in K$, which happens precisely when $\nu=\theta \mu^{-1}$ is a group cocycle.

Lemma 4.11. Let $Z$ be an abelian group, $K$ a group and $\theta: K \times K \rightarrow Z$ a cocycle such that $K \ltimes_{\theta} Z$ is a commutative A-loop. Let $\mu: K \times K \rightarrow Z$ be a group cocycle satisfying $\mu(x, y)=\mu(y, x)$ for every $x, y \in K$. Then $K \ltimes_{\mu \theta} Z$ is a commutative A-loop with the same (left) inner mappings as $K \ltimes_{\theta} Z$. 
Proof. Both $Q_{\theta}=K \ltimes_{\theta} Z, Q_{\mu \theta}=K \ltimes_{\mu \theta} Z$ are commutative loops. Since $\mu \theta \theta^{-1}$ is a group cocycle, $Q_{\mu \theta}$ has the same (left) inner mappings as $Q_{\theta}$, by Lemma 4.10, It therefore remains to show that every left inner mapping of $Q_{\mu \theta}$ is an automorphism.

Let $(x, a),(y, b) \in K \times Z$ and let $\varphi$ be a left inner mapping of $Q_{\mu \theta}$ (and hence of $Q_{\theta}$ ). Denote by · the multiplication in $Q_{\theta}$ and by $*$ the multiplication in $Q_{\mu \theta}$. Then

$$
\varphi((x, a) *(y, b))=\varphi((x, a) \cdot(y, b) \cdot(1, \mu(x, y)))=\varphi((x, a)) \cdot \varphi((y, b)) \cdot(1, \mu(x, y)),
$$

because $(1, \mu(x, y)) \in Z$ is a central element. The equation (4.5) in fact shows that $\varphi((x, a))=$ $\left(x, a^{\prime}\right)$ for some $a^{\prime}$, and similarly, $\varphi((y, b))=\left(y, b^{\prime}\right)$ for some $b^{\prime}$. Thus

$\varphi((x, a)) \cdot \varphi((y, b)) \cdot(1, \mu(x, y))=\left(x, a^{\prime}\right) \cdot\left(y, b^{\prime}\right) \cdot(1, \mu(x, y))=\left(x, a^{\prime}\right) *\left(y, b^{\prime}\right)=\varphi((x, a)) * \varphi((y, b))$, proving $\varphi \in \operatorname{Aut}\left(Q_{\mu \theta}\right)$.

\section{A Class of Commutative A-Loops of order $p^{3}$}

Let $Q$ be a commutative A-loop of odd order. Equivalently, let $Q$ be a finite commutative A-loop in which the mapping $x \mapsto x^{2}$ is a bijection of $Q$ (cf. [6, Lemma 3.1]). For $x \in Q$, denote by $x^{1 / 2}$ the unique element of $Q$ such that $\left(x^{1 / 2}\right)^{2}=x$. Define a new operation $\circ$ on $Q$ by

$$
x \circ y=\left(x^{-1} \backslash x y^{2}\right)^{1 / 2} .
$$

By [6, Lemma 3.5], $(Q, \circ)$ is a Bruck loop. By [6, Corollary 3.11], $(Q, \circ)$ is commutative if and only if it is isomorphic to $Q$.

Proposition 5.1. Let $p$ be an odd prime, and let $Q$ be a commutative A-loop of order $p, 2 p$, $4 p, p^{2}, 2 p^{2}$ or $4 p^{2}$. Then $Q$ is an abelian group.

Proof. Loops of order less than 5 are abelian groups. By the Decomposition Theorem mentioned in the introduction, it remains to prove that commutative A-loops of order $p$ and $p^{2}$ are abelian groups. For $|Q|=p$, this follows from the Lagrange Theorem and power-associativity. Assume that $|Q|=p^{2}$. Then $(Q, \circ)$ is a Bruck loop of order $p^{2}$, in particular a Bol loop of order $p^{2}$. Burn showed in [3] that all Bol loops of order $p^{2}$ are groups, and hence $(Q, \circ)$ is an abelian group. Consequently, $Q$ is an abelian group.

In this section we initiate the study of nonassociative commutative A-loops of order $p^{3}$. We conjecture that the class of loops constructed below accounts for all such loops.

Lemma 5.2. There is no commutative A-loop with center of prime index.

Proof. For a contradiction, let $Q$ be a commutative A-loop such that $|Q / Z(Q)|=p$ for some prime $p$. By Proposition 5.1, $Q / Z(Q)$ is the cyclic group of order $p$. Let $x \in Q \backslash Z(Q)$. Then $|x Z(Q)|=p$ and every element of $Q$ can be written as $x^{i} z$, where $0 \leq i<p$ and $z \in Z(Q)$. With $0 \leq i, j, k<p$ and $z_{1}, z_{2}, z_{3} \in Z(Q)$ we have

$$
\left(x^{i} z_{1} \cdot x^{j} z_{2}\right) \cdot x^{k} z_{3}=\left(x^{i} x^{j}\right) x^{k} \cdot z_{1} z_{2} z_{3}=x^{i}\left(x^{j} x^{k}\right) \cdot z_{1} z_{2} z_{3}=x^{i} z_{1} \cdot\left(x^{j} z_{2} \cdot x^{k} z_{3}\right)
$$

by power-associativity, so $Q$ is an abelian group with center of prime index, a contradiction.

Hence a nonassociative commutative A-loop of order $p^{3}$ has center of size 1 or $p$. (By the result announced in the introduction, we know, in fact, that the center must have size $p$ if $p$ is odd.)

Let $n \geq 1$. The overflow indicator is the function $(-,-)_{n}: \mathbb{Z}_{n} \times \mathbb{Z}_{n} \rightarrow\{0,1\}$ defined by

$$
(x, y)_{n}=\left\{\begin{array}{l}
1, \text { if } x+y \geq n \\
0, \text { otherwise }
\end{array}\right.
$$


Denote by $\oplus$ the addition in $\mathbb{Z}_{n}$, and note that for $x, y \in \mathbb{Z}_{n}$ we have $x \oplus y=x+y-n(x, y)_{n}$, and thus

$$
(x, y)_{n}=\frac{x+y-(x \oplus y)}{n} .
$$

Lemma 5.3. We have

$$
(x, y)_{n}+(x \oplus y, z)_{n}=(y, z)_{n}+(x, y \oplus z)_{n}
$$

for every $x, y, z \in \mathbb{Z}_{n}$.

Proof. Using (5.1), the identity (5.2) can be rewritten as

$$
x+y-(x \oplus y)+(x \oplus y)+z-(x \oplus y \oplus z)=y+z-(y \oplus z)+x+(y \oplus z)-(x \oplus y \oplus z),
$$

which holds.

From now on we write + for the addition in $\mathbb{Z}_{n}$, too.

For $n \geq 1$ and $a, b \in \mathbb{Z}_{n}$, define $\mathcal{Q}_{a, b}\left(\mathbb{Z}_{n}\right)$ on $\mathbb{Z}_{n} \times \mathbb{Z}_{n} \times \mathbb{Z}_{n}$ by

$$
\left(x_{1}, x_{2}, x_{3}\right)\left(y_{1}, y_{2}, y_{3}\right)=\left(x_{1}+y_{1}+\left(x_{2}+y_{2}\right) x_{3} y_{3}+a\left(x_{2}, y_{2}\right)_{n}+b\left(x_{3}, y_{3}\right)_{n}, x_{2}+y_{2}, x_{3}+y_{3}\right) \text {. }
$$

Then $\mathcal{Q}_{a, b}\left(\mathbb{Z}_{n}\right)$ can be seen as a central extension of $\mathbb{Z}_{n}$ by $\mathbb{Z}_{n} \times \mathbb{Z}_{n}$ via the loop cocycle $\theta\left(\left(x_{2}, x_{3}\right),\left(y_{2}, y_{3}\right)\right)=\left(x_{2}+y_{2}\right) x_{3} y_{3}+a\left(x_{2}, y_{2}\right)_{n}+b\left(x_{3}, y_{3}\right)_{n}$, and hence $\mathcal{Q}_{a, b}\left(\mathbb{Z}_{n}\right)$ is a commutative loop with neutral element $(0,0,0)$.

Note that we can write $\theta$ as $\theta=\mu+\nu$, where $\mu\left(\left(x_{2}, y_{2}\right),\left(x_{3}, y_{3}\right)\right)=\left(x_{2}+y_{2}\right) x_{3} y_{3}$ and $\nu\left(\left(x_{2}, y_{2}\right),\left(x_{3}, y_{3}\right)\right)=a\left(x_{2}, y_{2}\right)_{n}+b\left(x_{3}, y_{3}\right)_{n}$. By Lemma 5.3, $\nu$ is a group cocycle.

Proposition 5.4. Let $n \geq 2$ and $a, b \in \mathbb{Z}_{n}$. Let $Q=\mathcal{Q}_{a, b}\left(\mathbb{Z}_{n}\right)$ and $x=\left(x_{1}, x_{2}, x_{3}\right), y=$ $\left(y_{1}, y_{2}, y_{3}\right), z=\left(z_{1}, z_{2}, z_{3}\right) \in Q$. Then:

(i) $x \backslash y=\left(y_{1}-x_{1}-\left(y_{3}-x_{3}\right) x_{3} y_{2}-a\left(x_{2}, y_{2}-x_{2}\right)_{n}-b\left(x_{3}, y_{3}-x_{3}\right)_{n}, y_{2}-x_{2}, y_{3}-x_{3}\right)$,

(ii) $L_{y, x}(z)=x y \backslash x(y z)=\left(z_{1}+y_{3}\left(x_{3} z_{2}-x_{2} z_{3}\right), z_{2}, z_{3}\right)$,

(iii) $Q$ is a nonassociative commutative A-loop of order $n^{3}$,

(iv) $N_{\lambda}(Q)=Z(Q)=\mathbb{Z}_{n} \times 0 \times 0, N_{\mu}(Q)=\mathbb{Z}_{n} \times \mathbb{Z}_{n} \times 0$ as subsets of $Q$,

(v) $Q / Z(Q) \cong \operatorname{Inn}(Q) \cong \mathbb{Z}_{n} \times \mathbb{Z}_{n}$, and $\operatorname{Inn}(Q)=\left\{L_{u, v} ; u, v \in Q\right\}$,

(vi) for every $m \geq 0, x^{m}=\left(m x_{1}+2\left(\begin{array}{c}m+1 \\ 3\end{array}\right) x_{2} x_{3}^{2}+a t_{2}+b t_{3}, m x_{2}, m x_{3}\right)$, where $t_{i}=$ $\sum_{k=1}^{m-1}\left(x_{i}, k x_{i}\right)_{n}$. (As usual, the summation is considered empty and the binomial coefficient vanishes when $m<2$.)

Proof. Part (i) follows from the multiplication formula (5.3). Let $Q_{0}=\mathcal{Q}_{0,0}\left(\mathbb{Z}_{n}\right)$. By Lemma 4.10, it suffices to verify the formula (ii) for $Q_{0}$ instead of $Q$. Now, calculating in $Q_{0}$,

$x(y z)=\left(x_{1}+y_{1}+z_{1}+\left(y_{2}+z_{2}\right) y_{3} z_{3}+\left(x_{2}+y_{2}+z_{2}\right) x_{3}\left(y_{3}+z_{3}\right), x_{2}+y_{2}+z_{2}, x_{3}+y_{3}+z_{3}\right)$,

so (i) for $Q_{0}$ implies that $x y \backslash x(y z)$ is equal to

$\left(z_{1}+\left(y_{2}+z_{2}\right) y_{3} z_{3}+\left(x_{2}+y_{2}+z_{2}\right) x_{3}\left(y_{3}+z_{3}\right)-\left(x_{2}+y_{2}\right) x_{3} y_{3}-z_{3}\left(x_{3}+y_{3}\right)\left(x_{2}+y_{2}+z_{2}\right), z_{2}, z_{3}\right)$, which simplifies in a straightforward way to (ii).

By Lemma 4.11, to verify that left inner mappings of $Q$ are automorphisms of $Q$, it suffices to check that the left inner mappings of $Q_{0}$ are automorphisms of $Q_{0}$. With $u=\left(u_{1}, u_{2}, u_{3}\right)$, $v=\left(v_{1}, v_{2}, v_{3}\right)$, use (ii) to see that

$$
\begin{aligned}
& x y \backslash x(y u) \cdot x y \backslash x(y v) \\
& =\left(u_{1}+y_{3}\left(x_{3} u_{2}-x_{2} u_{3}\right), u_{2}, u_{3}\right)\left(v_{1}+y_{3}\left(x_{3} v_{2}-x_{2} v_{3}\right), v_{2}, v_{3}\right) \\
& =\left(u_{1}+v_{1}+y_{3}\left(x_{3}\left(u_{2}+v_{2}\right)-x_{2}\left(u_{3}+v_{3}\right)\right)+\left(u_{2}+v_{2}\right) u_{3} v_{3}+a\left(u_{2}, v_{2}\right)_{n}+b\left(u_{3}, v_{3}\right)_{n}, u_{2}+v_{2}, u_{3}+v_{3}\right) \\
& =x y \backslash x(y \cdot u v) .
\end{aligned}
$$

Hence $Q$ is a commutative A-loop of order $n^{3}$. 
To calculate the middle nucleus, we can once again resort to the loop $Q_{0}$, since the group cocycle will not play any role in identities that are consequences of associativity. We have

$$
\begin{aligned}
y \cdot\left(x_{1}, x_{2}, 0\right) z & =y\left(x_{1}+z_{1}, x_{2}+z_{2}, z_{3}\right) \\
& =\left(x_{1}+y_{1}+z_{1}+\left(x_{2}+y_{2}+z_{2}\right) y_{3} z_{3}, x_{2}+y_{2}+z_{2}, y_{3}+z_{3}\right) \\
& =\left(x_{1}+y_{1}, x_{2}+y_{2}, y_{3}\right) z=y\left(x_{1}, x_{2}, 0\right) \cdot z,
\end{aligned}
$$

so $\mathbb{Z}_{n} \times \mathbb{Z}_{n} \times 0 \leq N_{\mu}\left(Q_{0}\right)$. On the other hand,

$$
\left(0,0, x_{3}\right)\left(x_{1}, x_{2}, 0\right)=\left(x_{1}, x_{2}, x_{3}\right),
$$

so to prove that $\left(x_{1}, x_{2}, x_{3}\right) \notin N_{\mu}\left(Q_{0}\right)$ whenever $x_{3} \neq 0$, it suffices to show that $\left(0,0, x_{3}\right) \notin$ $N_{\mu}\left(Q_{0}\right)$ whenever $x_{3} \neq 0$. Now,

$$
\begin{aligned}
(0,0,1) \cdot\left(0,0, x_{3}\right)(0,1,0) & =(0,0,1)\left(0,1, x_{3}\right)=\left(x_{3}, 1,1+x_{3}\right) \\
\neq & \left(0,1,1+x_{3}\right)=\left(0,0,1+x_{3}\right)(0,1,0)=(0,0,1)\left(0,0, x_{3}\right) \cdot(0,1,0)
\end{aligned}
$$

shows just that. Similarly,

$$
\begin{aligned}
\left(x_{1}, 0,0\right) \cdot y z & =\left(x_{1}, 0,0\right)\left(y_{1}+z_{1}+\left(y_{2}+z_{2}\right) y_{3} z_{3}, y_{2}+z_{2}, y_{3}+z_{3}\right) \\
& =\left(x_{1}+y_{1}+z_{1}+\left(y_{2}+z_{2}\right) y_{3} z_{3}, y_{2}+z_{2}, y_{3}+z_{3}\right) \\
& =\left(x_{1}+y_{1}, y_{2}, y_{3}\right) z=\left(x_{1}, 0,0\right) y \cdot z
\end{aligned}
$$

proves that $\mathbb{Z}_{n} \times 0 \times 0 \leq N_{\lambda}\left(Q_{0}\right)$, and, for $x_{2} \neq 0$,

$$
\begin{aligned}
& \left(x_{1}, x_{2}, 0\right) \cdot(0,0,1)(0,0,1)=\left(x_{1}, x_{2}, 0\right)(0,0,2)=\left(x_{1}, x_{2}, 2\right) \\
& \quad \neq\left(x_{1}+x_{2}, x_{2}, 2\right)=\left(x_{1}, x_{2}, 1\right)(0,0,1)=\left(x_{1}, x_{2}, 0\right)(0,0,1) \cdot(0,0,1)
\end{aligned}
$$

implies that $N_{\lambda}(Q)=\mathbb{Z}_{n} \times 0 \times 0$ (recall that $N_{\lambda}(Q) \leq N_{\mu}(Q)$ in any A-loop $Q$ ).

Consider the mapping $\varphi: Q \rightarrow \operatorname{Inn}(Q)$ defined by

$$
\varphi\left(x_{1}, x_{2}, x_{3}\right)=L_{(0,0,1),\left(0, x_{2}, x_{3}\right)} .
$$

Then

$$
\begin{aligned}
& \varphi\left(x_{1}, x_{2}, x_{3}\right) \varphi\left(y_{1}, y_{2}, y_{3}\right)\left(z_{1}, z_{2}, z_{3}\right) \\
& =\varphi\left(x_{1}, x_{2}, x_{3}\right)\left(z_{1}+y_{3} z_{2}-y_{2} z_{3}, z_{2}, z_{3}\right)=\left(z_{1}+y_{3} z_{2}-y_{2} z_{3}+x_{3} z_{2}-x_{2} z_{3}, z_{2}, z_{3}\right) \\
& =\varphi\left(\left(x_{1}, x_{2}, x_{3}\right)\left(y_{1}, y_{2}, y_{3}\right)\right)\left(z_{1}, z_{2}, z_{3}\right)
\end{aligned}
$$

and $\varphi$ is a homomorphism. Its kernel consists of all $\left(x_{1}, x_{2}, x_{3}\right) \in Q$ such that $x_{3} z_{2}-x_{2} z_{3}=0$ for every $z_{2}, z_{3} \in Q$. Thus $\operatorname{ker} \varphi=\left\{\left(x_{1}, 0,0\right) ; x_{1} \in \mathbb{Z}_{n}\right\}$. To prove (v), it remains to show that $\varphi$ is onto $\operatorname{Inn}(Q)$. By (ii),

$$
L_{\left(y_{1}, y_{2}, y_{3}\right),\left(x_{1}, x_{2}, x_{3}\right)}=L_{\left(0,0, y_{3}\right),\left(0, x_{2}, x_{3}\right)}=L_{(0,0,1),\left(0, y_{3} x_{2}, y_{3} x_{3}\right)} .
$$

This means that $\operatorname{Im} \varphi$ contains a generating subset of $\operatorname{Inn}(Q)$, and hence it is equal to $\operatorname{Inn}(Q)$. In fact, purely on the grounds of cardinality, we have $\operatorname{Inn}(Q)=\left\{L_{u, v} ; u, v \in Q\right\}$.

The identity of (vi) clearly holds when $m=0$. Assume that it holds for some $m \geq 0$. Let $t_{i}^{m}=\sum_{k=1}^{m}\left(x_{i}, k x_{i}\right)_{n}$. By power-associativity, we have

$$
\begin{aligned}
x^{m+1} & =x x^{m}=x\left(m x_{1}+2\left(\begin{array}{c}
m+1 \\
3
\end{array}\right) x_{2} x_{3}^{2}+a t_{2}^{m-1}+b t_{3}^{m-1}, m x_{2}, m x_{3}\right) \\
& =\left((m+1) x_{1}+2\left(\begin{array}{c}
m+1 \\
3
\end{array}\right) x_{2} x_{3}^{2}+(m+1) x_{2} m x_{3}^{2}+a t_{2}^{m}+b t_{3}^{m},(m+1) x_{2},(m+1) x_{3}\right),
\end{aligned}
$$

Since $2\left(\begin{array}{c}m+1 \\ 3\end{array}\right)+(m+1) m=2\left(\begin{array}{c}m+2 \\ 3\end{array}\right)$, we are through.

Lemma 5.5. Let $p$ be a prime and $a, b \in \mathbb{Z}_{p}$. Let $Q=\mathcal{Q}_{a, b}\left(\mathbb{Z}_{p}\right)$. Then: 
(i) if $(a, b)=(0,0)$ and $p \neq 3$ then $Q$ has exponent $p$,

(ii) if $(a, b) \neq(0,0)$ or $p=3$ then $Q$ has exponent $p^{2}$,

(iii) if $a=0$ then $N_{\mu}(Q) \cong \mathbb{Z}_{p} \times \mathbb{Z}_{p}$,

(iv) if $a \neq 0$ then $N_{\mu}(Q) \cong \mathbb{Z}_{p^{2}}$.

Proof. By [6], every element of $Q$ has order a power of $p$, so $Q$ has exponent $p, p^{2}$ or $p^{3}$. Since $Q$ is nonassociative by Proposition [5.4 the exponent is either $p$ or $p^{2}$.

Assume that $(a, b)=(0,0)$. Then by Proposition 5.4(vi),

$$
\left(x_{1}, x_{2}, x_{3}\right)^{p}=\left(2\left(\begin{array}{c}
p+1 \\
3
\end{array}\right) x_{2} x_{3}^{2}, 0,0\right) .
$$

The integer $2\left(\begin{array}{c}p+1 \\ 3\end{array}\right)$ is divisible by $p$ if and only if $p \neq 3$. This proves (i).

To show (ii), it remains to prove that $Q$ has exponent $p^{2}$ if $(a, b) \neq(0,0)$. Assume that $a \neq 0$, and note that, by Proposition 5.4(vi),

$$
(0,1,0)^{p}=\left(a \sum_{k=1}^{p-1}(1, k)_{p}, 0,0\right)=\left(a(1, p-1)_{p}, 0,0\right)=(a, 0,0) .
$$

This means that $Q$ does not have exponent $p$, and it also shows, by Proposition 5.4(iv), that $N_{\mu}(Q) \cong \mathbb{Z}_{p^{2}}$. Similarly, when $b \neq 0$, use

$$
(0,0,1)^{p}=\left(b \sum_{k=1}^{p-1}(1, k)_{p}, 0,0\right)=(b, 0,0)
$$

to conclude that $Q$ does not have exponent $p$.

Finally, when $a=0$, we have $\left(x_{1}, x_{2}, 0\right)^{p}=0$ by Proposition 5.4 (vi), so $N_{\mu}(Q) \cong \mathbb{Z}_{p} \times \mathbb{Z}_{p}$ by Proposition 5.4(iv).

As usual, denote by $\mathbb{Z}_{n}^{*}$ the set of all invertible elements of $\mathbb{Z}_{n}$.

Lemma 5.6. Let $n>0$. If $b, c \in \mathbb{Z}_{n}^{*}$ then $\mathcal{Q}_{0, b}\left(\mathbb{Z}_{n}\right) \cong \mathcal{Q}_{0, c}\left(\mathbb{Z}_{n}\right)$.

Proof. Define $\varphi: \mathcal{Q}_{0, b}\left(\mathbb{Z}_{n}\right) \rightarrow \mathcal{Q}_{0, c}\left(\mathbb{Z}_{n}\right)$ by $\left(x_{1}, x_{2}, x_{3}\right) \mapsto\left((c / b) x_{1},(c / b) x_{2}, x_{3}\right)$, and note that $\varphi$ is a bijection since $b, c$ are invertible.

Denote by $\cdot$ the multiplication in $\mathcal{Q}_{0, b}\left(\mathbb{Z}_{n}\right)$ and by $*$ the multiplication in $\mathcal{Q}_{0, c}\left(\mathbb{Z}_{n}\right)$. Then

$$
\begin{aligned}
& \varphi\left(\left(x_{1}, x_{2}, x_{3}\right) \cdot\left(y_{1}, y_{2}, y_{3}\right)\right)=\varphi\left(\left(x_{1}+y_{1}+\left(x_{2}+y_{2}\right) x_{3} y_{3}+b\left(x_{3}, y_{3}\right)_{n}, x_{2}+y_{2}, x_{3}+y_{3}\right)\right) \\
& =\left(\frac{c}{b}\left(x_{1}+y_{1}+\left(x_{2}+y_{2}\right) x_{3} y_{3}+b\left(x_{3}, y_{3}\right)_{n}\right), \frac{c}{b}\left(x_{2}+y_{2}\right), x_{3}+y_{3}\right) \\
& =\left(\frac{c}{b} x_{1}, \frac{c}{b} x_{2}, x_{3}\right) *\left(\frac{c}{b} y_{1}, \frac{c}{b} y_{2}, y_{3}\right)=\varphi\left(\left(x_{1}, x_{2}, x_{3}\right)\right) * \varphi\left(\left(y_{1}, y_{2}, y_{3}\right)\right) .
\end{aligned}
$$

Let $p$ be an odd prime. Recall that $a \in \mathbb{Z}_{p}^{*}$ is a quadratic residue modulo $p$ if there is $x \in \mathbb{Z}_{p}^{*}$ such that $x^{2} \equiv a(\bmod p)$. Else $a$ is a quadratic nonresidue modulo $p$. Also recall that $a b^{-1}$ is a quadratic residue if and only if either both $a, b$ are quadratic residues or both $a, b$ are quadratic nonresidues.

Lemma 5.7. Let $p$ be an odd prime and $a_{1}, a_{2} \in \mathbb{Z}_{p}^{*}$. If $a_{1}, a_{2}$ are either both quadratic residues or both quadratic nonresidues then $\mathcal{Q}_{a_{1}, 0}\left(\mathbb{Z}_{p}\right) \cong \mathcal{Q}_{a_{2}, 0}\left(\mathbb{Z}_{p}\right)$. 
Proof. Since $a_{1} a_{2}^{-1}$ is a quadratic residue, there is $u$ such that $a_{2}=a_{1} u^{2}$. Define $\varphi$ : $\mathcal{Q}_{a_{1}, 0}\left(\mathbb{Z}_{p}\right) \rightarrow \mathcal{Q}_{a_{2}, 0}\left(\mathbb{Z}_{p}\right)$ by $\left(x_{1}, x_{2}, x_{3}\right) \mapsto\left(u^{2} x_{1}, x_{2}, u x_{3}\right)$. Then $\varphi$ is a bijection. Denote by $\cdot$ the multiplication in $\mathcal{Q}_{a_{1}, 0}\left(\mathbb{Z}_{p}\right)$ and by $*$ the multiplication in $\mathcal{Q}_{a_{2}, 0}\left(\mathbb{Z}_{p}\right)$. Then

$$
\begin{aligned}
& \varphi\left(\left(x_{1}, x_{2}, x_{3}\right) \cdot\left(y_{1}, y_{2}, y_{3}\right)\right)=\varphi\left(\left(x_{1}+y_{1}+\left(x_{2}+y_{2}\right) x_{3} y_{3}+a_{1}\left(x_{2}, y_{2}\right)_{p}, x_{2}+y_{2}, x_{3}+y_{3}\right)\right) \\
& =\left(u^{2}\left(x_{1}+y_{1}+\left(x_{2}+y_{2}\right) x_{3} y_{3}+a_{1}\left(x_{2}, y_{2}\right)_{p}\right), x_{2}+y_{2}, u\left(x_{3}+y_{3}\right)\right) \\
& =\left(u^{2} x_{1}+u^{2} y_{1}+\left(x_{2}+y_{2}\right) u x_{3} u y_{3}+a_{2}\left(x_{2}, y_{2}\right)_{p}, x_{2}+y_{2}, u\left(x_{3}+y_{3}\right)\right) \\
& =\left(u^{2} x_{1}, x_{2}, u x_{3}\right) *\left(u^{2} y_{1}, y_{2}, u y_{3}\right)=\varphi\left(\left(x_{1}, x_{2}, x_{3}\right)\right) * \varphi\left(\left(y_{1}, y_{2}, y_{3}\right)\right) .
\end{aligned}
$$

Lemma 5.8. For a prime $p$, let $Q_{1}=\mathcal{Q}_{a, b}\left(\mathbb{Z}_{p}\right)=\left(Q_{1}, \cdot\right), Q_{2}=\mathcal{Q}_{a, c}\left(\mathbb{Z}_{p}\right)=\left(Q_{2}, *\right)$ and let $f: Q_{1} \rightarrow Q_{2}$ be an isomorphism that pointwise fixes the middle nucleus of $Q_{1}$ (i.e., $f$ is identical on $\left.\mathbb{Z}_{p} \times \mathbb{Z}_{p} \times 0\right)$. Then there are $A, B \in \mathbb{Z}_{p}$ and $C \in \mathbb{Z}_{p}^{*}$ such that

$$
f\left(x_{1}, x_{2}, x_{3}\right)=\left(x_{1}, x_{2}, 0\right) *(A, B, C)^{x_{3}}
$$

for every $\left(x_{1}, x_{2}, x_{3}\right) \in Q_{1}$.

In addition, every mapping $f: Q_{1} \rightarrow Q_{2}$ defined by (5.4) with $A, B \in \mathbb{Z}_{p}$ and $C \in \mathbb{Z}_{p}^{*}$ is a bijection that pointwise fixes $N_{\mu}\left(Q_{1}\right)$.

Proof. Let $f: Q_{1} \rightarrow Q_{2}$ be an isomorphism that pointwise fixes $N_{\mu}\left(Q_{1}\right)$. As $Q_{1} / N_{\mu}\left(Q_{1}\right)$ is a cyclic group, $f$ is determined by the image of any element in $Q_{1} \backslash N_{\mu}\left(Q_{1}\right)$. Let $f(0,0,1)=$ $(A, B, C)$. We must have $C \neq 0$, else $f$ is not a bijection. Since $\left(x_{1}, x_{2}, x_{3}\right)=\left(x_{1}, x_{2}, 0\right)\left(0,0, x_{3}\right)$ and $\left(0,0, x_{3}\right)=(0,0,1)^{x_{3}}$ by Proposition 5.4(vi), we have

$$
f\left(x_{1}, x_{2}, x_{3}\right)=f\left(x_{1}, x_{2}, 0\right) * f(0,0,1)^{x_{3}}=\left(x_{1}, x_{2}, 0\right) *(A, B, C)^{x_{3}} .
$$

Conversely, define $f: Q_{1} \rightarrow Q_{2}$ by (5.4), where $C \neq 0$. Then $f$ obviously pointwise fixes $N_{\mu}\left(Q_{1}\right)$. To show that $f$ is a bijection, assume that $f\left(x_{1}, x_{2}, x_{3}\right)=f\left(y_{1}, y_{2}, y_{3}\right)$. Since the last coordinate of $\left(x_{1}, x_{2}, 0\right) *(A, B, C)^{x_{3}}$ is $C x_{3}$, we conclude that $x_{3}=y_{3}$. The second coordinate of $\left(x_{1}, x_{2}, 0\right) *(A, B, C)^{x_{3}}$ is $x_{2}+B x_{3}$, and we conclude that $x_{2}=y_{2}$. Then $x_{1}=y_{1}$ follows from the multiplication formula for $Q_{2}$ and from Proposition 5.4(vi).

Lemma 5.9. Let $p \neq 3$ be a prime and assume that $a, b, c \in \mathbb{Z}_{p}$ are such that $a+c \equiv b$ $(\bmod p)$. Let $Q_{1}=\mathcal{Q}_{a, b}\left(\mathbb{Z}_{p}\right)=\left(Q_{1}, \cdot\right)$ and $Q_{2}=\mathcal{Q}_{a, c}\left(\mathbb{Z}_{p}\right)=\left(Q_{2}, *\right)$. Then $f: Q_{1} \rightarrow Q_{2}$ defined by (5.4) with $(A, B, C)=(0,1,1)$ is an isomorphism.

Proof. For $x \in \mathbb{Z}_{p}$, let $x^{\prime}=(x-1) x(x+1) / 3$. By Lemma [5.8, $f$ is a bijection onto $Q_{2}$ that pointwise fixes $N_{\mu}\left(Q_{1}\right)$. Upon expanding the formula (5.4), we see that

$$
f\left(x_{1}, x_{2}, x_{3}\right)=\left(x_{1}+x_{3}^{\prime}+a\left(x_{2}, x_{3}\right)_{p}, x_{2}+x_{3}, x_{3}\right),
$$

since the expression $\sum_{k=1}^{x_{3}-1}(1, k)_{p}$ vanishes for every $x_{3}<p$. Let

$$
\left(u_{1}, u_{2}, u_{3}\right)=f\left(x_{1}, x_{2}, x_{3}\right) * f\left(y_{1}, y_{2}, y_{3}\right)
$$

and

$$
\left(v_{1}, v_{2}, v_{3}\right)=f\left(\left(x_{1}, x_{2}, x_{3}\right) \cdot\left(y_{1}, y_{2}, y_{3}\right)\right) .
$$

A quick calculation then shows that

$$
\left(u_{2}, u_{3}\right)=\left(v_{2}, v_{3}\right)=\left(x_{2}+x_{3}+y_{2}+y_{3}, x_{3}+y_{3}\right),
$$

$u_{1}$ is equal to

$$
x_{1}+x_{3}^{\prime}+a\left(x_{2}, x_{3}\right)_{p}+y_{1}+y_{3}^{\prime}+a\left(y_{2}, y_{3}\right)_{p}+\left(x_{2}+x_{3}+y_{2}+y_{3}\right) x_{3} y_{3}+a\left(x_{2}+x_{3}, y_{2}+y_{3}\right)_{p}+c\left(x_{3}, y_{3}\right)_{p},
$$


while $v_{1}$ is equal to

$$
x_{1}+y_{1}+\left(x_{2}+y_{2}\right) x_{3} y_{3}+a\left(x_{2}, y_{2}\right)_{p}+b\left(x_{3}, y_{3}\right)_{p}+\left(x_{3}+y_{3}\right)^{\prime}+a\left(x_{2}+y_{2}, x_{3}+y_{3}\right)_{p} .
$$

Now, $x_{3}^{\prime}+y_{3}^{\prime}=\left(x_{2}+y_{2}\right) x_{3} y_{3}+\left(x_{3}+y_{3}\right)^{\prime}$. Using (5.1), it is easy to see that

$$
\left(x_{2}, x_{3}\right)_{p}+\left(y_{2}, y_{3}\right)_{p}+\left(x_{2}+x_{3}, y_{2}+y_{3}\right)_{p}=\left(x_{2}, y_{2}\right)_{p}+\left(x_{2}+y_{2}, x_{3}+y_{3}\right)_{p}+\left(x_{3}, y_{3}\right)_{p} .
$$

Hence we are done by $a+c \equiv b(\bmod p)$.

Corollary 5.10. Let $p \neq 3$ be a prime, $a \in \mathbb{Z}_{p}^{*}$ and $b, c \in \mathbb{Z}_{p}$. Then $\mathcal{Q}_{a, b}\left(\mathbb{Z}_{p}\right)$ is isomorphic to $\mathcal{Q}_{a, c}\left(\mathbb{Z}_{p}\right)$.

Proof. By Lemma 5.9 we have $\mathcal{Q}_{a, 0}\left(\mathbb{Z}_{p}\right) \cong \mathcal{Q}_{a, a}\left(\mathbb{Z}_{p}\right) \cong \mathcal{Q}_{a, 2 a}\left(\mathbb{Z}_{p}\right)$, and so on.

5.1. Ring construction. Note that for $a=b=0$, the construction (5.3) makes sense over any commutative ring $R$, not just over $\mathbb{Z}_{n}$. We can summarize the most important features of the construction as follows:

Proposition 5.11. Let $R \neq 0$ be a commutative ring. Let $Q=\mathcal{Q}(R)$ be defined on $R \times R \times R$ by

$$
\left(x_{1}, x_{2}, x_{3}\right)\left(y_{1}, y_{2}, y_{3}\right)=\left(x_{1}+y_{1}+\left(y_{2}+x_{2}\right) x_{3} y_{3}, x_{2}+y_{2}, x_{3}+y_{3}\right) .
$$

Then $Q$ is a commutative A-loop satisfying $N_{\lambda}(Q)=Z(Q)=R \times 0 \times 0$ and $N_{\mu}(Q)=R \times R \times 0$.

Proof. See the relevant parts of the proof of Proposition 5.4.

5.2. Towards the classification of commutative A-loops of order $p^{3}$. The results obtained up to this point come close to describing the isomorphism types of all loops $\mathcal{Q}_{a, b}\left(\mathbb{Z}_{p}\right)$ for all primes $p \neq 3$.

Fix $p \neq 3$. The loop $\mathcal{Q}_{0,0}\left(\mathbb{Z}_{p}\right)$ is of exponent $p$ and is not isomorphic to any other loop $\mathcal{Q}_{a, b}\left(\mathbb{Z}_{p}\right)$, by Lemma 5.5. By Lemma 5.5 and Corollary 5.10, the loops $\left\{\mathcal{Q}_{0, b}\left(\mathbb{Z}_{p}\right) ; 0<b<p\right\}$ form an isomorphism class. By Lemmas [5.7 and [5.8, each of the two sets $I_{r}=\left\{\mathcal{Q}_{a, b}\left(\mathbb{Z}_{p}\right) ; a>0\right.$ is a quadratic residue modulo $p$ and $0 \leq b<p\}$ and $I_{n}=\left\{\mathcal{Q}_{a, b}\left(\mathbb{Z}_{p}\right) ; a>0\right.$ is a quadratic nonresidue modulo $p$ and $0 \leq b<p\}$ consist of pairwise isomorphic loops.

However, we did not manage to establish the following:

Conjecture 5.12. Let $p>3$ be a prime, let $a_{1} \in \mathbb{Z}_{p}^{*}$ be a quadratic residue and $a_{2} \in \mathbb{Z}_{p}^{*}$ be a quadratic nonresidue. Then $\mathcal{Q}_{a_{1}, 0}\left(\mathbb{Z}_{p}\right)$ is not isomorphic to $\mathcal{Q}_{a_{2}, 0}\left(\mathbb{Z}_{p}\right)$.

We have verified the conjecture computationally with the GAP [5] package LOOPS [8] for $p=5,7$. It appears that one of the distinguishing isomorphism invariants is the multiplication group $\operatorname{Mlt}(Q)=\left\langle L_{x}, R_{x} ; x \in Q\right\rangle$.

The loops $\mathcal{Q}_{a, b}\left(\mathbb{Z}_{p}\right)$ behave differently for $p=3$ due to the fact that 3 is the only prime $p$ for which $p$ does not divide $2\left(\begin{array}{c}p+1 \\ 3\end{array}\right)$. Denote by $f_{(A, B, C)}$ the bijection defined by (5.4). It can be verified by computer that $f_{(0,1,1)}$ is an exceptional isomorphism $\mathcal{Q}_{0,0}\left(\mathbb{Z}_{3}\right) \rightarrow \mathcal{Q}_{0,1}\left(\mathbb{Z}_{3}\right), f_{(0,0,2)}$ is an isomorphism $\mathcal{Q}_{1,1}\left(\mathbb{Z}_{3}\right) \rightarrow \mathcal{Q}_{1,2}\left(\mathbb{Z}_{3}\right), f_{(0,1,2)}$ is an isomorphism $\mathcal{Q}_{2,0}\left(\mathbb{Z}_{3}\right) \rightarrow \mathcal{Q}_{2,1}\left(\mathbb{Z}_{3}\right)$ and $f_{(0,1,1)}$ is an isomorphism $\mathcal{Q}_{2,0}\left(\mathbb{Z}_{3}\right) \rightarrow \mathcal{Q}_{2,2}\left(\mathbb{Z}_{3}\right)$. The loops $\mathcal{Q}_{0,0}\left(\mathbb{Z}_{3}\right), \mathcal{Q}_{1,0}\left(\mathbb{Z}_{3}\right), \mathcal{Q}_{1,1}\left(\mathbb{Z}_{3}\right)$ and $\mathcal{Q}_{2,0}\left(\mathbb{Z}_{3}\right)$ contain precisely $12,6,24$ and 18 elements of order 9 , respectively, so no two of them are isomorphic.

Altogether, Figure 1 depicts the isomorphism classes of loops $\mathcal{Q}_{a, b}\left(\mathbb{Z}_{p}\right)$ as connected components, for $p \in\{2,3,5,7\}$ and $a, b \in \mathbb{Z}_{p}$. Moreover, if Conjecture [5.12 is true, the pattern established by $p=2,5$ and 7 continues for all primes $p>7$.

It is reasonable to ask whether, for an odd prime $p$, there are nonassociative commutative A-loops of order $p^{3}$ not of the form $\mathcal{Q}_{a, b}\left(\mathbb{Z}_{p}\right)$.

Using a linear-algebraic approach to cocycles (see Subsection 6.4), we managed to classify all nonassociative commutative A-loops of order $p^{3}$ with nontrivial center, for $p \in\{2,3,5,7\}$. 


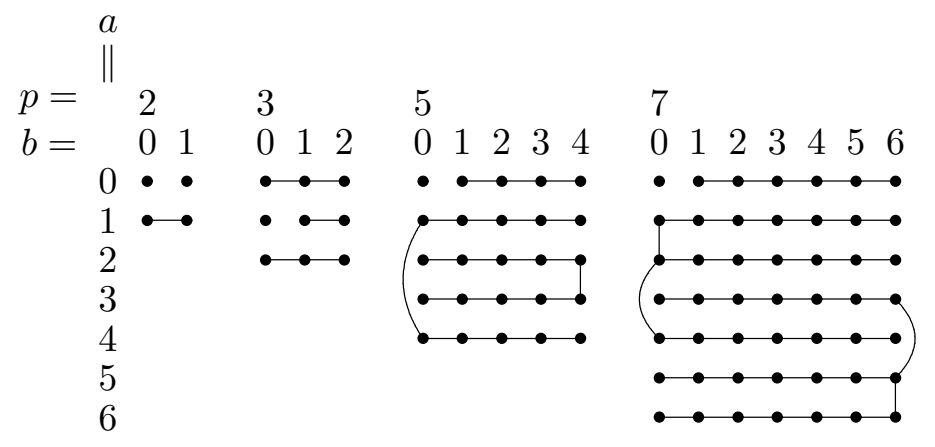

FiguRE 1. Isomorphism classes of loops $\mathcal{Q}_{a, b}\left(\mathbb{Z}_{p}\right)$ for $p \in\{2,3,5,7\}$.

TABle 1. Commutative A-loops up to isomorphism (up to isotopism).

\begin{tabular}{r||c|c|c|c|c|c|c|c}
$n$ & 8 & 15 & 16 & 21 & 24 & 27 & 30 & 32 \\
\hline \hline commutative groups & 3 & 1 & 5 & 1 & 3 & 3 & 1 & 7 \\
\hline commutative nonassociative A-loops & $4(3)$ & 1 & $46(38)$ & 1 & $4(3)$ & 4 & 1 & $?$ \\
\hline $\begin{array}{r}\text { commutative nonassociative A-loops } \\
\text { with nontrivial center }\end{array}$ & $3(2)$ & 0 & $44(37)$ & 0 & $4(3)$ & 4 & 1 & $?$ \\
\hline $\begin{array}{r}\text { commutative nonassociative A-loops } \\
\text { of exponent } p\end{array}$ & 2 & - & $12(11)$ & - & - & 0 & - & $?$ \\
\hline $\begin{array}{r}\text { commutative nonassociative A-loops } \\
\text { of exponent } p\end{array}$ & 1 & - & 10 & - & - & 0 & - & $211(210)$ \\
$\begin{array}{r}\text { with nontrivial center } \\
\hline\end{array}$ & & & & & & &
\end{tabular}

It turns out that all such loops are of the type $\mathcal{Q}_{a, b}\left(\mathbb{Z}_{p}\right)$. In particular, $p=3$ is the only prime for which there is no nonassociative commutative A-loop of order $p^{3}$ and exponent $p$.

Problem 5.13. Let $p$ be an odd prime and $Q$ a nonassociative commutative A-loop of order $p^{3}$. Is $Q$ isomorphic to $\mathcal{Q}_{a, b}\left(\mathbb{Z}_{p}\right)$ for some $a, b \in \mathbb{Z}_{p}$ ?

\section{ENUMERATion}

We believe that future work will benefit from an enumeration of small commutative A-loops. The results are summarized in Table 1, which lists all orders $n \leq 32$ for which there exists a nonassociative commutative A-loop.

If there is only one number in a cell of the table, it is both the number of isomorphism classes and the number of isotopism classes. If there are two numbers in a cell, the first one is the number of isomorphism classes and the second one (in parentheses) is the number of isotopism classes.

All computations were done with the finite model builder Mace4 and with the GAP package LOOPS on a Unix machine with a single $2 \mathrm{GHz}$ processor, with computational times for individual orders ranging from seconds to hours.

6.1. Comments on commutative A-loops of order 8. For classification up to isomorphism, see Section 3 . 
Lemma 6.1. Let $G$ be a commutative loop, $g \in \operatorname{Aut}(G)$, and let $t_{1}$, $t_{2}$ be fixed points of $g$. Define $f_{i}(x)=g(x) t_{i}$, for $i=1$, 2. If there is $z \in G$ such that $g(z)=z^{-1} t_{1}^{-1} t_{2}$, then $G\left(f_{1}\right)$, $G\left(f_{2}\right)$ are isotopic.

Proof. Denote by $*$ the multiplication in $G\left(f_{1}\right)$ and by o the multiplication in $G\left(f_{2}\right)$. For $x \in G$, define $\alpha(x)=x, \alpha(\bar{x})=\overline{x z^{-1}}, \beta(x)=z x, \beta(\bar{x})=\bar{x}, \gamma(x)=z x$, and $\gamma(\bar{x})=\bar{x}$. Then

$$
\begin{aligned}
& \alpha(x) \circ \beta(y)=x \circ z y=x z y=\gamma(x y)=\gamma(x * y), \\
& \alpha(x) \circ \beta(\bar{y})=x \circ \bar{y}=\overline{x y}=\gamma(\overline{x y})=\gamma(x * \bar{y}), \\
& \alpha(\bar{x}) \circ \beta(y)=\overline{x z^{-1}} \circ z y=\overline{x y}=\gamma(\overline{x y})=\gamma(\bar{x} * y), \\
& \alpha(\bar{x}) \circ \beta(\bar{y})=\overline{x z^{-1}} \circ \bar{y}=g\left(x z^{-1} y\right) t_{2}=z g(x y) t_{1}=\gamma\left(g(x y) t_{1}\right)=\gamma(\bar{x} * \bar{y}),
\end{aligned}
$$

where we have used $g(z)=z^{-1} t_{1}^{-1} t_{2}$ in the last line.

Let $G=\mathbb{Z}_{2} \times \mathbb{Z}_{2}=\langle a\rangle \times\langle b\rangle$ be the Klein group. Consider the transposition $g=(a, b)$ with fixed points $t_{1}=1, t_{2}=a b$. Let $f_{i}(x)=g(x) t_{i}$, for $i=1,2$. Then $b=g(a)=a^{-1} t_{1}^{-1} t_{2}$, so $G\left(f_{1}\right), G\left(f_{2}\right)$ are isotopic by Lemma 6.1 .

\subsection{Comments on commutative A-loops of order 15 and 21.}

Lemma 6.2. Let $Q$ be a nonassociative commutative A-loop of order $p_{0} p_{1}$, where $p_{0} \neq p_{1}$ are odd primes. Then there is $0 \leq i \leq 1$ such that $Q$ contains a normal subloop $S$ of order $p_{i}$, and all elements in $Q \backslash S$ have order $p_{i+1}$, where the subscript is calculated modulo 2.

Proof. We will use results of [6] mentioned in the introduction without further reference. Since $Q$ is of odd order, it is solvable. Since $Q$ is also nonassociative, there is a normal subloop $S$ of $Q$ such that $1 \neq S \neq Q$. By the Lagrange Theorem, $|S|=p_{i}$ for some $0 \leq i \leq 1$. Without loss of generality, let $|S|=p_{0}$. Let $y \in Q \backslash S$ and let $T$ be the preimage of the subloop $\langle y S\rangle$ of $Q / S$. By the Lagrange Theorem again, $y^{p_{1}}=1$, as the only other alternative $|y|=p_{0} p_{1}$ would mean that $Q$ is a group by power-associativity.

The information afforded by Lemma 6.2 is sufficient to construct all nonassociative commutative A-loops of order 15 and 21 by the finite model builder Mace4. It turns out that in each case there is a unique such loop. These two loops were constructed already by Drápal [4, Proposition 3.1]. Nevertheless the following problem remains open:

Problem 6.3. Classify commutative A-loops of order $p q$, where $p<q$ are odd primes.

We have some reasons to believe that there is no nonassociative commutative A-loop of order 35 .

6.3. Comments on commutative A-loops of order 16. Among the 12 nonassociative commutative A-loops of order 16 and exponent 2, three have inner mapping groups of orders that are not a power of 2, namely 12,56 and 56. We now construct the two nonassociative commutative A-loops of order 16 and exponent 2 with inner mapping groups of order 56, and we show that they are isotopic.

Let $G=\mathbb{Z}_{4} \times \mathbb{Z}_{2}$. Define $g \in \operatorname{Aut}(G)$ by $g(i, j)=(i, i+j \bmod 2)$. Note that $t_{1}=$ $(0,0), t_{2}=(2,1)$ are fixed points of $g$, and let $f_{i}(x)=g(x)+t_{i}$. Then $G\left(f_{1}\right), G\left(f_{2}\right)$ are the two announced loops, and they are isotopic by Lemma 6.1 since $g(1,0)=(1,1)$ and $-(1,0)-(0,0)+(2,1)=(1,1)$. 


\subsection{Comments on commutative A-loops of order 32 and exponent 2 with nontrivial}

center. The methods developed in [9] in order to classify Moufang loops of order 64 can be adopted to other classes of loops. Using the cocycle formula of Corollary 4.1 and the classification of commutative A-loops of order 16 from Subsection 6.3, we were able to classify all commutative A-loops of order 32 and of exponent 2 with nontrivial center.

We now briefly describe the search, following the method of [9] closely. For more details, see $[9$.

Let $Q$ be a commutative A-loop of order 32 and exponent 2 with nontrivial center. Then $Z(Q)$ is obviously an elementary abelian 2-group, and hence it possesses a 2-element central subgroup $Z=(Z,+, 0)$. Then $Q / Z=K$ is a commutative A-loop of order 16 and exponent 2 .

The loop cocycles $\theta: K \times K \rightarrow Z$ form a vector space $V$ over $Z=\mathrm{GF}(2)$ with respect to addition $(\theta+\mu)(x, y)=\theta(x, y)+\mu(x, y)$. The vector space $V$ has basis $\left\{\theta_{u, v} ; 1 \neq u \in K, 1 \neq\right.$ $v \in K\}$, where

$$
\theta_{u, v}(x, y)= \begin{cases}1, & \text { if }(u, v)=(x, y) \\ 0, & \text { otherwise. }\end{cases}
$$

The extension $K \ltimes_{\theta} Z$ will be a commutative A-loop of exponent 2 if and only if $\theta$ belongs to the subspace $C=\{\theta \in V ; \theta$ satisfies (4.1), $\theta(x, x)=0$ for every $x \in K$ and $\theta(x, y)=\theta(y, x)$ for every $x, y \in K\}$.

For every $x, y, z, x^{\prime} \in K$, the equation (4.1) can be viewed as a linear equation over $\mathrm{GF}(2)$ in variables $\theta_{u, v}$. Similarly, for every $x, y \in K$ we obtain linear equations from the condition $\theta(x, y)=\theta(y, x)$, and from $\theta(x, x)=0$.

Upon solving this system of linear equations, we obtain (a basis of) $C$, and it is in principle possible to construct all extensions $K \ltimes_{\theta} Z$ for $\theta \in C$. The two computational problems we face are: (i) the dimension of $C$ can be large, (ii) it is costly to sort the resulting loops up to isomorphism. In order to overcome these obstacles, we take advantage of coboundaries and of an induced action of $\operatorname{Aut}(K)$ on $C$.

Let $\tau: K \times Z$ be a mapping satisfying $\tau(1)=0$. Then $\delta \tau: K \times K \rightarrow Z$ defined by

$$
\delta \tau(x, y)=\tau(x y)-\tau(x)-\tau(y)
$$

is a coboundary. Coboundaries form a subspace $B$ of $V$.

In fact, $B$ is a subspace of $C$. This can be proved explicitly by verifying that every coboundary $\theta=\delta \tau$ satisfies the identity (4.1), $\theta(x, y)=\theta(y, x)$ and $\theta(x, x)=0$. The verification of (4.1) is a bit unpleasant, so it is worth realizing that every coboundary $\theta$ satisfies the group cocycle identity

$$
\theta(x, y)+\theta(x y, z)=\theta(y, x)+\theta(x, y z)
$$

and hence also any cocycle identity that follows from associativity, in particular (4.1).

Moreover, if $\theta, \mu: K \times K \rightarrow Z$ are two cocycles such that $\theta-\mu$ is a coboundary, then $K \ltimes_{\theta} Z$ is isomorphic to $K \ltimes_{\theta} Z$, cf. [9, Lemma 9]. It therefore suffices to construct loops $K \ltimes_{\theta} Z$, where $\theta \in D, C=B \oplus D$.

Given $\theta \in V$ and $\varphi \in \operatorname{Aut}(K)$, we define $\theta_{\varphi} \in V$ by

$$
\theta_{\varphi}(x, y)=\theta(\varphi(x), \varphi(y)) .
$$

This action of $\operatorname{Aut}(K)$ on $V$ induces an action on $D$. Moreover, by [9, Lemma 14], $K \ltimes_{\theta} Z$ is isomorphic to $K \ltimes_{\theta_{\varphi}} Z$. It therefore suffices to construct loops $K \ltimes_{\theta} Z$, where we take one $\theta$ from each orbit of $\operatorname{Aut}(K)$ on $D$. 
Using each of the 13 commutative A-loops of order 16 and exponent 2 as $K$ (the elementary abelian group of order 16 must also be taken into account), the above search finds 355 commutative A-loops of order 32 and exponent 2 within several minutes. The final isomorphism search takes several hours with LOOPS.

The lone isotopism $\mathbb{Z}_{2} \times Q_{1} \rightarrow \mathbb{Z}_{2} \times Q_{2}$ is induced by the isotopism $Q_{1} \rightarrow Q_{2}$ described in Subsection 6.3.

\section{Acknowledgement}

We thank the anonymous referee for the nice proof of Lemma 4.4 .

\section{REFERENCES}

[1] R. H. Bruck, A survey of binary systems, third printing, corrected, Ergebnisse der Mathematik und Ihrer Grenzgebiete, new series, volume 20, Springer, 1971.

[2] R. H. Bruck and L. J. Paige, Loops whose inner mappings are automorphisms, Ann. of Math. (2) 63 (1956), 308-323.

[3] R. P. Burn, Finite Bol loops, Math. Proc. Cambridge Philos. Soc. 84 (1978), no. 3, 377-385.

[4] A. Drápal, A class of commutative loops with metacyclic inner mapping groups, submitted.

[5] The GAP Group, GAP - Groups, Algorithms, and Programming, Version 4.4; 2004, http://www .gap-system.org.

[6] P. Jedlička, M. K. Kinyon and P. Vojtěchovský, The structure of commutative automorphic loops, to appear in Trans. Amer. Math. Soc.

[7] W. McCune, Prover9 and Mace4, http://www.prover9.org.

[8] G. P. Nagy and P. Vojtěchovský, LOOPS: Computing with quasigroups and loops, version 2.0.0, package for GAP, http://www . math.du.edu/loops.

[9] G. P. Nagy and P. Vojtěchovský, The Moufang loops of order 64 and 81, J. Symbolic Computation 42 (2007), no. 9, 871-883.

[10] H. O. Pflugfelder, Quasigroups and Loops: Introduction, Sigma Series in Pure Mathematics 7, Heldermann Verlag Berlin, 1990.

(Jedlička) Department of Mathematics, Faculty of Engineering, Czech University of Life Sciences, Kamýcká 129, 16521 Prague 6-Suchdol, Czech Republic

E-mail address, Jedlička: jedlickap@tf.czu.cz

(Kinyon) Department of Mathematics, University of Denver, 2360 S Gaylord St, Denver, ColORADO, 80208, U.S.A.

E-mail address, Kinyon: mkinyon@math.du.edu

(Vojtěchovský) Department of Mathematics, University of Denver, 2360 S Gaylord St, Denver, Colorado, 80208, U.S.A.

E-mail address, Vojtěchovský: petr@math.du.edu 